\title{
Interfacial Assembly Directed Unique Mesoporous Architectures: From Symmetric to Asymmetric
}

\author{
Tiancong Zhao, Liang Chen, Runfeng Lin, Pengfei Zhang, Kun Lan, Wei Zhang, Xiaomin Li,* \\ and Dongyuan Zhao*
}

Cite This: Acc. Mater. Res. 2020, 1, 100-114

Read Online

CONSPECTUS: Functional mesoporous materials have experienced flourishing developments over the past 30 years, and they are now a big family including large variety of compositions and architectures. Due to large surface area, high pore volume, tunable pore size, and unique mesostructures, mesoporous nanomaterials have been widely used in catalysis, energy conversion and storage, drug delivery, etc. Out of the various methods of architecting mesoporous materials, the soft-templating method that allows regulating the architecture by tuning the assembly process, is used mostly for fabricating mesoporous materials. In soft templating methods, amphiphilic surfactants and precursors assemble into "soft" micelles, and micelles further assemble into mesoporous materials.

Usually, micelles homogeneously nucleate and assemble into mesoporous nanomaterials. Such a homogeneous assembly process naturally leads to "routine" structures such as nanospheres or other symmetric architectures. Introduction of nanoparticles during the micelle assembly process results in conventional core@shell structures, which though combining the mesoporous shell and functional core, is still simple. Nowadays, growing demands in diverse applications urgently require mesoporous nanomaterials with novelty in mesostructures, compositions, surface properties, morphologies, functionalities, etc. Therefore, researchers have been seeking to gain better control of the micelles' assembly process, pursuing the synthesis of mesoporous nanomaterials with unique architectures, especially morphology, mesostructure, surface chemistry, etc. Recently, the effect of "interface" during micelles' assembly process has aroused great attention. The introduction of an interface into the reaction system turns the micelles' homogeneous assembly into a heterogeneous one, altering their assembly behavior and thus leading to mesoporous nanomaterials with unique architectures. By manipulation of the interfacial-assembly behavior of micelles, a series of mesoporous nanomaterials with unique architectures have been developed, with structures ranging from symmetric to asymmetric. In this Account, the recent progresses in interfacial-assembly-directed mesoporous nanomaterials with unique architectures, both the fabrication and applications are systematic reviewed. The architectures of the obtained mesoporous nanomaterials are categorized into symmetric and asymmetric, including virus-like, bouquet-like, multishell hollow structures, and also Janus, multipods, hemisphere structures, etc. We introduced how interfaces such as nanoparticle-surface, water-oil interface, macropore scaffold interfaces, etc. are utilized to direct the assembly of micelles. The methods of controlling micelles' interfacial-assembly behavior, such as biphasic assembly method, interfacial-energy method, interface tension method, and so on are highlighted. The applications of these unique mesoporous nanomaterials in fields of catalysis, energy conversion, and biomedicine are surveyed. At the end of the review, a brief summary of the field, as well as shortcomings, challenges, and perspectives of the field are also included.

\section{INTRODUCTION}

Mesoporous materials have experienced flourishing developments over the past 30 years. ${ }^{1,2}$ The concept of "mesopores" means pores with a diameter in the range of $2-50 \mathrm{~nm}$, and nanoparticles containing mesopores are called mesoporous nanoparticles. Mesoporous silica is first reported in 1992, ${ }^{3}$ whereafter, in 1998, Zhao et al. proposed the evaporation induced self-assembly (EISA) method, in which triblock copolymers $\left(\mathrm{PEO}_{n}-\mathrm{PPO}_{m}-\mathrm{PEO}_{n}\right)$ were used to fabricate a series of mesoporous silica with large pore size, highly ordered and good stability, greatly promoting the development of mesoporous materials. ${ }^{4}$ At first the fabricated mesoporous materials are bulk, then the growing demands in application fields bring about nanosized mesoporous nanoparticles. ${ }^{2,5-8}$ To date, mesoporous nanomaterials are now a big family including a large variety of compositions and architectures. Due to large surface area, high pore volume, tunable pore size

Received: August 16, 2020

Revised: September 29, 2020

Published: October 7, 2020

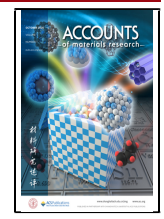



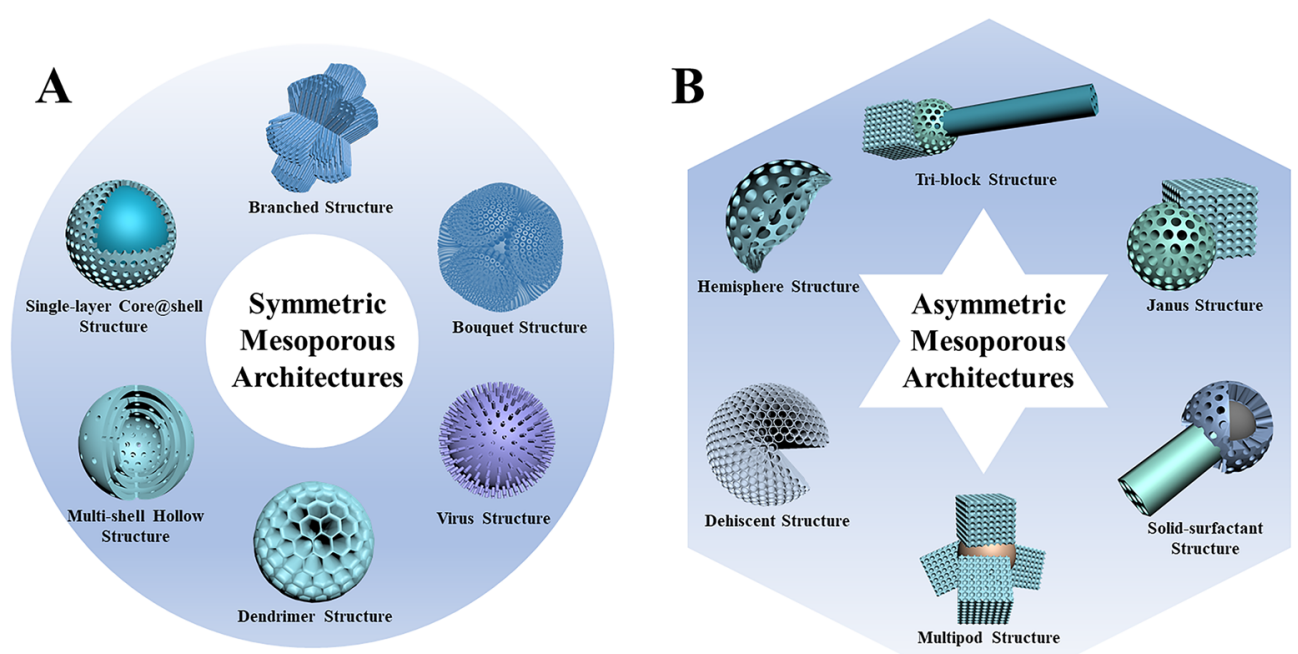

Figure 1. Overview of mesoporous nanomaterials with various unique architectures. Schematic illustration of mesoporous nanomaterials with (A) symmetric and (B) asymmetric architectures fabricated through interfacial-assembly strategy.

and unique mesostructures, mesoporous nanomaterials have been widely used in catalysis, ${ }^{9}$ energy conversion and storage, ${ }^{10-12}$ drug delivery, ${ }^{13}$ etc. Generally, the methods for the synthesis of order mesoporous materials can be classified as hard-templating and soft-templating routes. ${ }^{1,8,14}$ The hardtemplating route involves nanocasting by using presynthesized mesoporous solids or colloidal crystals as a sacrificial mold, which tends to be costly, time-consuming and inflexible in tuning mesostructures. The soft-templating route, which surfactant molecules and precursors coassemble into ordered mesostructured composites, allows regulating the architecture by tuning the assembly process. Therefore, the soft-templating route is much favored.

Besides homogeneous nucleation and growth for the synthesis of the conventional mesoporous nanomaterials, the introduction of interfaces turns the micelles' assembly process into a heterogeneous one, greatly enriching the structure and functionality of the obtained mesoporous nanomaterials. The most typical interfacial assembly is using nanoparticle's surface as interfaces, the micelles uniformly assemble on the surface of the nanoparticles, leading to core@shell structures. Yet, growing demands in diverse applications urgently require mesoporous nanomaterials with novelty in mesostructures, compositions, surface properties, morphologies, functionalities, etc. Therefore, researchers have been seeking to gain better control of the micelles' interfacial-assembly process, enabling the synthesis of mesoporous nanomaterials with unique architectures. Thus, it would be much favored that we can systemically review the methods for fabricating mesoporous nanomaterials with unique architectures, drawing out intrinsic connections between them, providing theoretical guidance for possible readers.

In this Account, we provide a systematic review on our groups' recent progresses in interfacial assembly directed mesoporous nanomaterials with unique architectures. The architecture of these mesoporous nanomaterials ranges from symmetric to asymmetric, including virus-like, ${ }^{15}$ bouquetlike, ${ }^{16}$ multishell hollow structures, ${ }^{17}$ and also Janus, ${ }^{18}$ multipods, ${ }^{19}$ hemisphere structures, ${ }^{20}$ etc. (Figure 1). The symmetric mesoporous nanomaterials are classified on the basis of the interface utilized during micelle assembly, such as nanoparticle-surface, solid-liquid interface, macropore scaffold interfaces, etc., are utilized to direct the assembly of micelles. The asymmetric mesoporous nanomaterials are, on the other hand, categorized using the methods of controlling micelles' interfacial-assembly behavior, such as biphasic assembly method, ${ }^{21}$ interfacial-energy method, ${ }^{18}$ interface tension method, ${ }^{20}$ and so on. The applications of these mesoporous materials in fields of catalysis, ${ }^{22,23}$ energy storage and conversion, ${ }^{11}$ biomedicine ${ }^{13}$ are surveyed. At the end of the review, challenges and perspectives of the field are also included.

\section{INTERFACIAL-ASSEMBLY-DIRECTED SYMMETRIC MESOPOROUS NANOMATERIALS}

Surfactant/precursor micelles usually self-assemble into spherical structures due to minimal energy, or specific morphologies based on the mesostructures (cubic leads to nanocubes, two-dimensional hexagonal leads to rods). ${ }^{2}$ For example, the assembly of silane oligomers hydrolyzed from tetraethyl orthosilicate (TEOS) and hexadecyltrimethylammonium bromide $(\mathrm{CTAB})$ in alkaline aqueous environment results in mesoporous silica nanoparticles. ${ }^{24}$ Mesoporous polymer nanoparticles can be obtained by assembling Pluronic F127 $\left(\mathrm{PEO}_{100}-\mathrm{PPO}_{65}-\mathrm{PEO}_{100}\right)$ with low molecular weight resorcinol formaldehyde resin (RF) precursors in a water and ethanol mixture. ${ }^{25,26}$ Mesoporous $\mathrm{TiO}_{2}$ microspheres with single-crystal-like anatase pore walls are also obtained by heating a gel of Pluronic $\mathrm{F} 127 / \mathrm{TiO}_{2}$-oligomer micelles at 70 ${ }^{\circ} \mathrm{C} .{ }^{27}$ These processes are conventional homogeneous micelleassembly processes. The structures of the obtained mesoporous nanomaterials are relatively simple.

When interfaces are introduced, the assembly behavior of micelles is turned from homogeneous assembly into heterogeneous interfacial assembly, leading to brand new architectures. ${ }^{14}$ The interfaces include solid-liquid, liquid-liquid, solid-solid interfaces, and so on. Manipulation of interfacial assembly allows the fabrication of unique mesoporous architectures with critically controlled mesostructures or unprecedented multilevel architectures. 


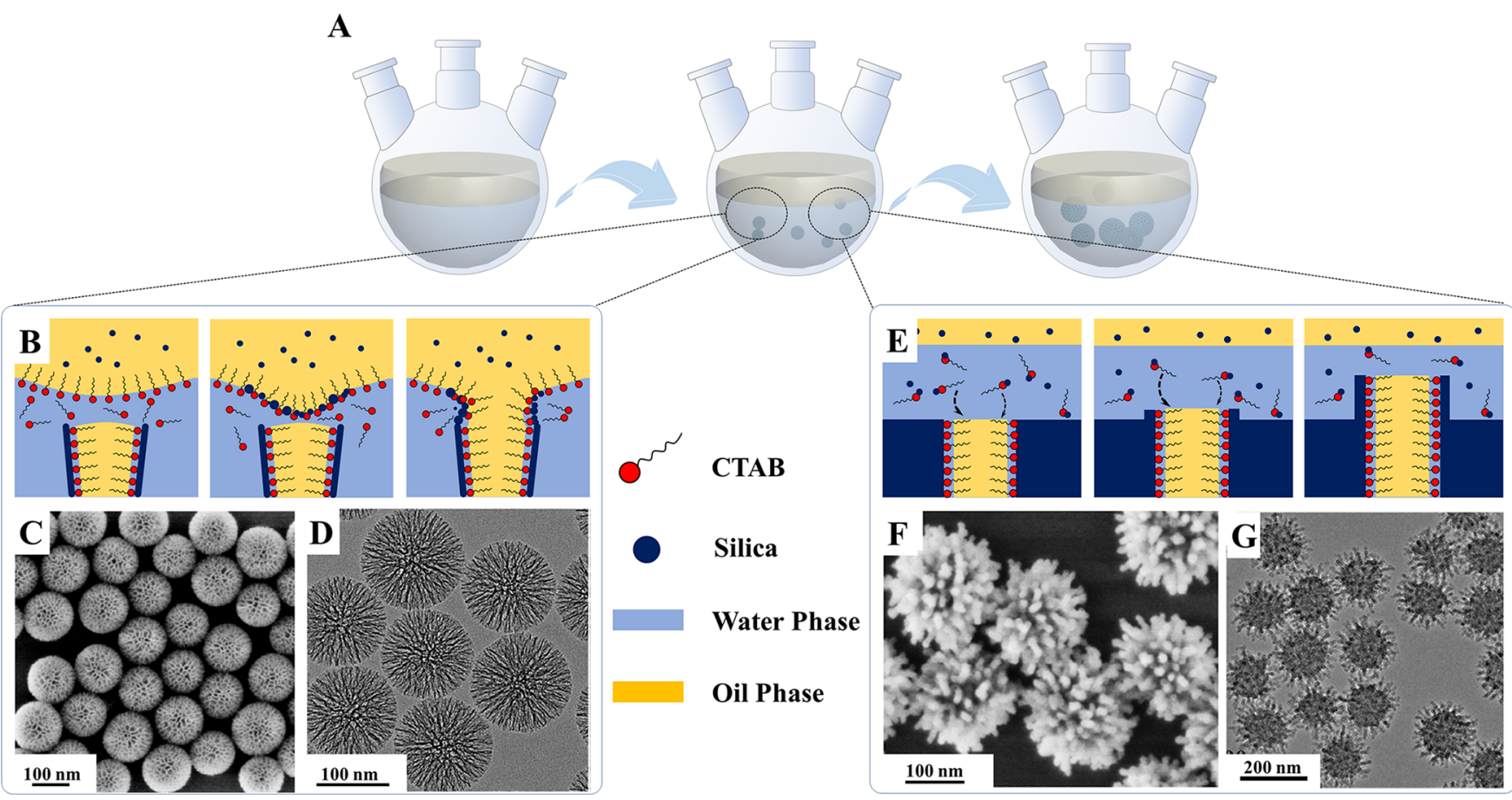

Figure 2. Uniform mesoporous silica nanomaterials synthesized through a water-oil interfacial assembly. (A) Stratified biphasic interfacial assembly process. (B) $-(\mathrm{G})$ Schematic illustration and TEM and SEM images of the mesoporous silica nanoparticles prepared through biphasic interfacial-assembly method: (B) - (D) dendrimer-like mesoporous silica nanoparticles and (E) -(G) virus-like mesoporous silica nanoparticles. Reproduced with permission from refs 15 and 21. Copyright 2017 and 2014 American Chemical Society.

\subsection{Liquid-Liquid Interfacial Assembly}

The liquid-liquid interface formed between two immiscible liquids is one of the most common interfaces in the wetchemistry synthesis, which can be used for the manipulation of the assembly of micelles. Shen et al. introduced an oil upperphase to conventional homogeneous aqueous reaction system, thus forming a stratified system and biphasic interfaces (Figure 2A). ${ }^{21}$ Surfactant $\mathrm{CTAB}$ and alkali triethanolamine are added in the water phase, and TEOS is introduced into the upper cyclohexane oil phase. TEOS can gradually diffuse to the oilwater interface, be hydrolyzed and assembled with surfactant $\mathrm{CTAB}$ into CTAB/silica-oligomer micelles. The assembly of micelles at the interface results in a "funneling" gradient assembly and growth process (Figure 2B), obtaining dendrimer-like mesoporous silica nanoparticles with diameters of $\sim 250 \mathrm{~nm}$ and large mesopores. The micelles' interfacialassembly manner can be tuned by varying the type and amount of oil phase as well as the concentration of silane, resulting in dendrimer-like mesoporous silica nanoparticles with very uniform pore size ranging from 3 to $15 \mathrm{~nm}$ (Figure 2C,D). Yue et al. found that the assembly of micelles at this biphasic interface could also be tuned with assistance of shearing force (stirring speed) ${ }^{28}$ With stirring speed tuned from 170 to 500 $\mathrm{rpm}$, the pore size can be further increased to $20 \mathrm{~nm}$. These works pave the way for architecting mesoporous silica nanoparticles with large pore sizes, widening their application potentials.

Wang et al. have further developed, also on the basis of the biphasic interfacial assembly, an epitaxial growth method for never-before-seen virus-like mesoporous nanoparticles (Figure $2 \mathrm{E}) .{ }^{15}$ In this reaction system, dendrimer-like mesoporous silica nanoparticles are first formed through isotropic growth process. Then, as the surfactant concentration is gradually decreased, the transformation of assembly behavior of micelles from isotropic radial assembly to single-micelle epitaxial assembly is induced. Thus, the short silica nanotubes are epitaxially grown from the mesochannels of the preformed dendrimer-like mesoporous silica nanoparticles. By varying the reaction parameters, 3D virus-like nanoparticles with welltuned inner core diameters $(60-160 \mathrm{~nm})$ and outer tubular lengths $(6-70 \mathrm{~nm})$ are successfully obtained (Figure $2 \mathrm{~F}, \mathrm{G}$ ).

Besides the stratified biphase that water and oil are separated with macroscopic interfaces, emulsion systems that water and oil are mixed with abundant microscopic water-oil interfaces are also used. Peng et al. have used a versatile nanoemulsion assembly approach, in which 1,3,5-trimethylbenzene (TMB) was used to mediate the interfacial-assembly behavior of Pluronic F127/PDA-oligomer $(\mathrm{PDA}=$ polydopamine $)$ micelles. Mesoporous PDA nanoparticles with uniform diameters $(\sim 145 \mathrm{~nm})$ and large, tunable pore sizes $(5-37$ $\mathrm{nm})$ can be generated. ${ }^{29}$ In this work, large yet unstable F127/ TMB/PDA nanoemulsion droplets are first formed, which can be merged and fused in the radial direction of the preformed PDA frameworks. Generally, PDA prefers to cross-link along the interfaces between the ultralarge nanoemulsions, thus forming the mesoporous PDA nanoparticles with open and accessible dendritic mesopores. This work demonstrates how organic molecules (TMB) significantly affect the interaction between surfactants and precursors during the interfacialassembly process.

Besides the liquid-liquid interface between the oil and water, the interface between ethanol and high viscosity glycerol is also used to confine the assembly of micelles for the synthesis of two-dimensional (2D) mesoporous $\mathrm{TiO}_{2}$ nanosheets. ${ }^{30}$ In this approach, Pluronic $\mathrm{F} 127 / \mathrm{TiO}_{2}$-oligomer micelles are first formed by evaporating an acid-THF solvent containing F127 and tetrabutyl titanate at $40{ }^{\circ} \mathrm{C}$. Then the 
A

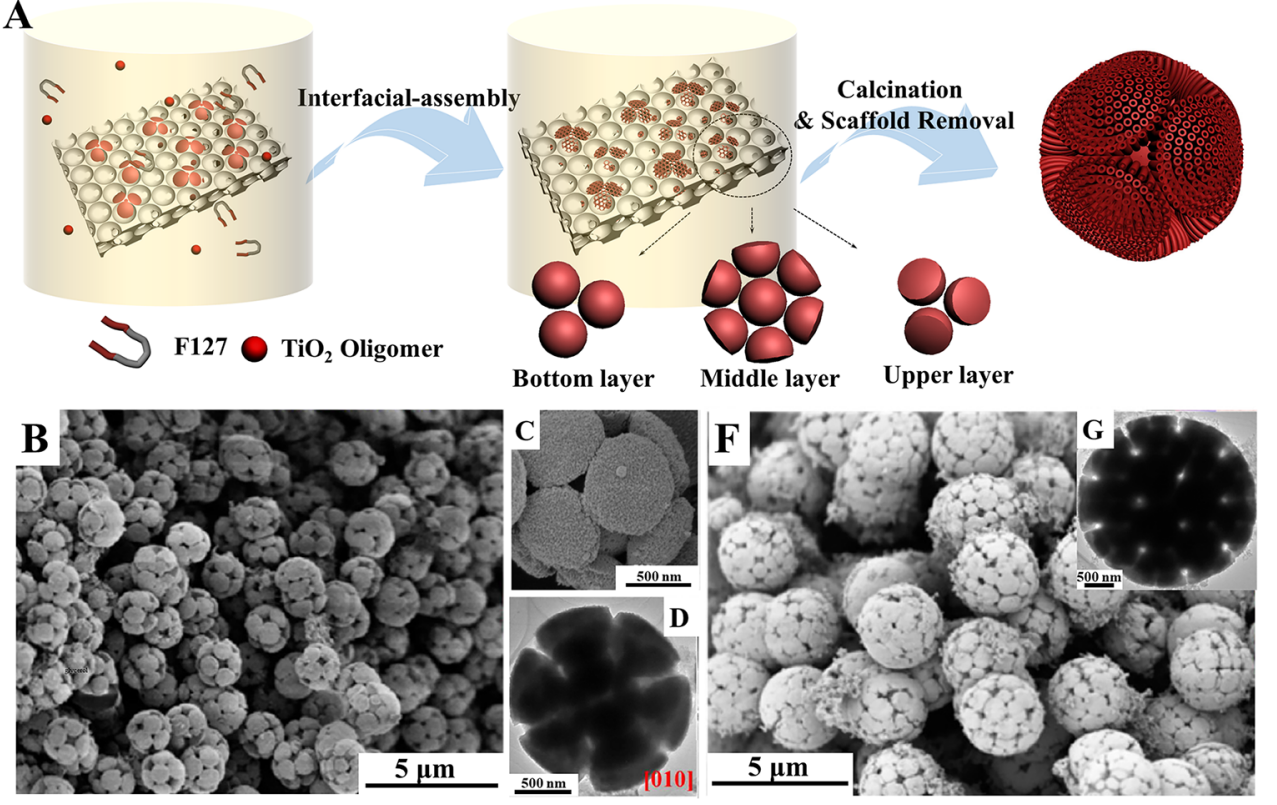

Figure 3. 3D Scaffold induced solid-liquid interfacial assembly for mesoporous $\mathrm{TiO}_{2}$ superstructures. Schematic illustration and TEM and SEM images with different magnifications of mesoporous $\mathrm{TiO}_{2}$ nanoparticles superstructures with unique (A) -(D) level-1 bouquet-like and (F, G) level2 bouquet-like architectures. Reproduced with permission from ref 16. Copyright 2017 American Chemical Society.

micelles are dissolved and heated to assemble in a mixture of ethanol and glycerol. Highly viscous glycerol confines the micelles so that they assemble in a $2 \mathrm{D}$ manner. This unique $2 \mathrm{D}$ interfacial assembly of micelles at this liquid-liquid interface results in the formation of ordered mesoporous $\mathrm{TiO}_{2}$ nanosheets, with which the thickness is fine-tuned from a single layer $(\sim 5 \mathrm{~nm})$ to multiple layers $(\sim 20 \mathrm{~nm})$ by changing the amount of glycerol. This template-free synthesis of $2 \mathrm{D}$ nanosheets through interfacial assembly opens up a brand-new territory of architecting unique mesoporous nanomaterials.

\subsection{Solid-Liquid Interfacial Assembly}

As another useful interface, the solid-liquid interface has been widely used in the synthesis of the core@shell structured mesoporous nanomaterials. ${ }^{31-33}$ Different from the simple solid core nanoparticles, the assembly of micelles can mimic the solid-interfaces to form the mesoporous nanomaterials with advanced 3D hierarchical architectures. Liu and Lan et al. assembled Pluronic F127/ $\mathrm{TiO}_{2}$-oligomer micelles within the confinement of a $3 \mathrm{D}$ ordered macroporous carbon (3DOMC) scaffold. Interestingly, 3D mesoporous $\mathrm{TiO}_{2}$ superstructures with well-defined bouquet-posy-like topologies are obtained (Figure 3A). ${ }^{16}$ Due to the confinement of 3DOMC, micelles are assembled on the interface of macropores, and the mesoporous $\mathrm{TiO}_{2}$ microspheres are forged according to the shape of the scaffolds. Mesoporous $\mathrm{TiO}_{2}$ superstructures with a spherical core and 12 symmetric satellite hemispheres can be obtained. The mesoporous channels in superstructured mesoporous $\mathrm{TiO}_{2}$ are radially distributed and highly interconnected (Figure 3B-D). The architectural complexity is further increased by tuning the macroporous size of the 3DOMC or the amount of impregnated $\mathrm{TiO}_{2}$ precursors. Typically, when the size of $\mathrm{TiO}_{2}$ microspheres increases to $\sim 3.5 \mu \mathrm{m}$, a more complex 3D hierarchical mesoporous architecture with 13 spherical cores and up to 44 symmetric satellite hemispheres is achieved (Figure 3F,G). Wang et al. have also utilized the 3DOMC to tune the assembly of silica based micells. ${ }^{34}$ In compared with the homogeneous assembly of $\mathrm{P} 123 /$ silica-oligomer $\left(\mathrm{P} 123=\mathrm{PEO}_{20}-\mathrm{PPO}_{70}-\mathrm{EO}_{20}\right)$ micelles to form the rod-shaped SBA-15, the confinement of the $3 \mathrm{DOMC}$ solid interfaces forces the micelles to assemble into spherical architectures, resulting in $350 \mathrm{~nm} \mathrm{SBA-15}$ nanoparticles with ordered concentric circled mesoporous channels.

Besides scaffolds, nanocrystals may also provide facets as solid interfaces to modulate the assembly of micelles. Zhang et al. synthesized branched mesoporous $\mathrm{TiO}_{2}$ mesocrystals. ${ }^{35}$ $\mathrm{MoS}_{2}-\mathrm{CdS}$ nanocrystals are introduced into the reaction system, which mediate the formation of crystalline $\mathrm{TiO}_{2}$ clusters. The crystalline surfaces of the $\mathrm{TiO}_{2}$ clusters can further direct the epitaxial assembly of micelles in the solution along the $\langle 001\rangle,\langle 101\rangle$, and $\langle 111\rangle$ directions, leading to branched mesoporous $\mathrm{TiO}_{2}$ mesocrystals. Due to the crystallization-driven epitaxial assembly, these nanomaterials possess both unique branched architectures as well as coherent atomic domains, providing great application potentials.

Micelles usually can assemble homogeneously on the surfaces of nanoparticles, forming a smooth and uniform mesoporous layer. Interestingly, Yue et al. synthesized cauliflower-shaped $\mathrm{Fe}_{3} \mathrm{O}_{4} @ \mathrm{RF} @ \mathrm{mSiO}_{2} \quad(\mathrm{RF}=$ resorcinol formaldehyde resin) nanoparticles with tunable surface roughness through a kinetics-controlled interface coassembly and deposition of CTAB/silica-oligomer micelles on the $\mathrm{Fe}_{3} \mathrm{O}_{4} @$ RF. $^{36}$ The surface roughness and morphology, i.e., the mesoporous silica domain size on the RF surface, can be well tuned by kinetics-controlled interface coating process via adjusting the amount of basic catalysts. This robust solidliquid interfacial-engineering enables a controllable nucleation of $\mathrm{CTAB} /$ silica-oligomer micelles on RF.

The assembly of micelles on solid interfaces follows a kinetic mediated manner and can be described using a LaMer curve: the concentration of micelle-precursor composites rises and surpasses the critical nucleation concentration for precursors' heterogeneous nucleation on the interfaces to start. Nucleation consumes and lowers the precursor concentration, and then 


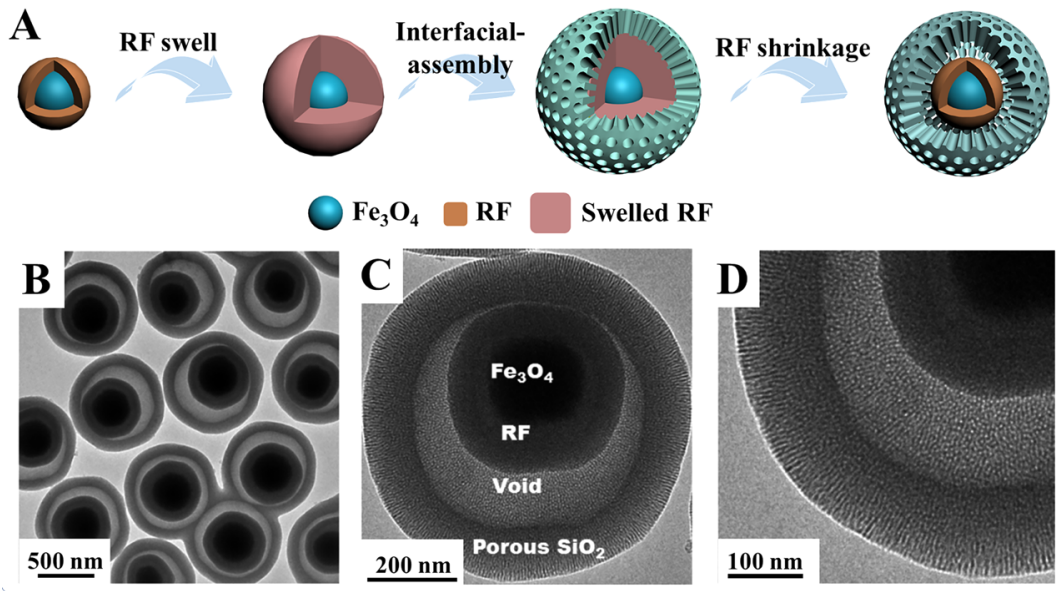

Figure 4. Manipulation of the solid-solid interfaces for one-pot synthesis of the yolk@shell nanostructures. (A) Schematic illustrations and (B)(D) TEM images with different magnifications of the novel one-pot synthesized $\mathrm{Fe}_{3} \mathrm{O}_{4} @ \mathrm{RF} @ \mathrm{mSiO}_{2}\left(\mathrm{RF}=\right.$ resorcinol formaldehyde resin, $\mathrm{mSiO}_{2}$ = mesoporous silica) yolk@shell architecture. Reproduced with permission from ref 37. Copyright 2017 American Chemical Society.

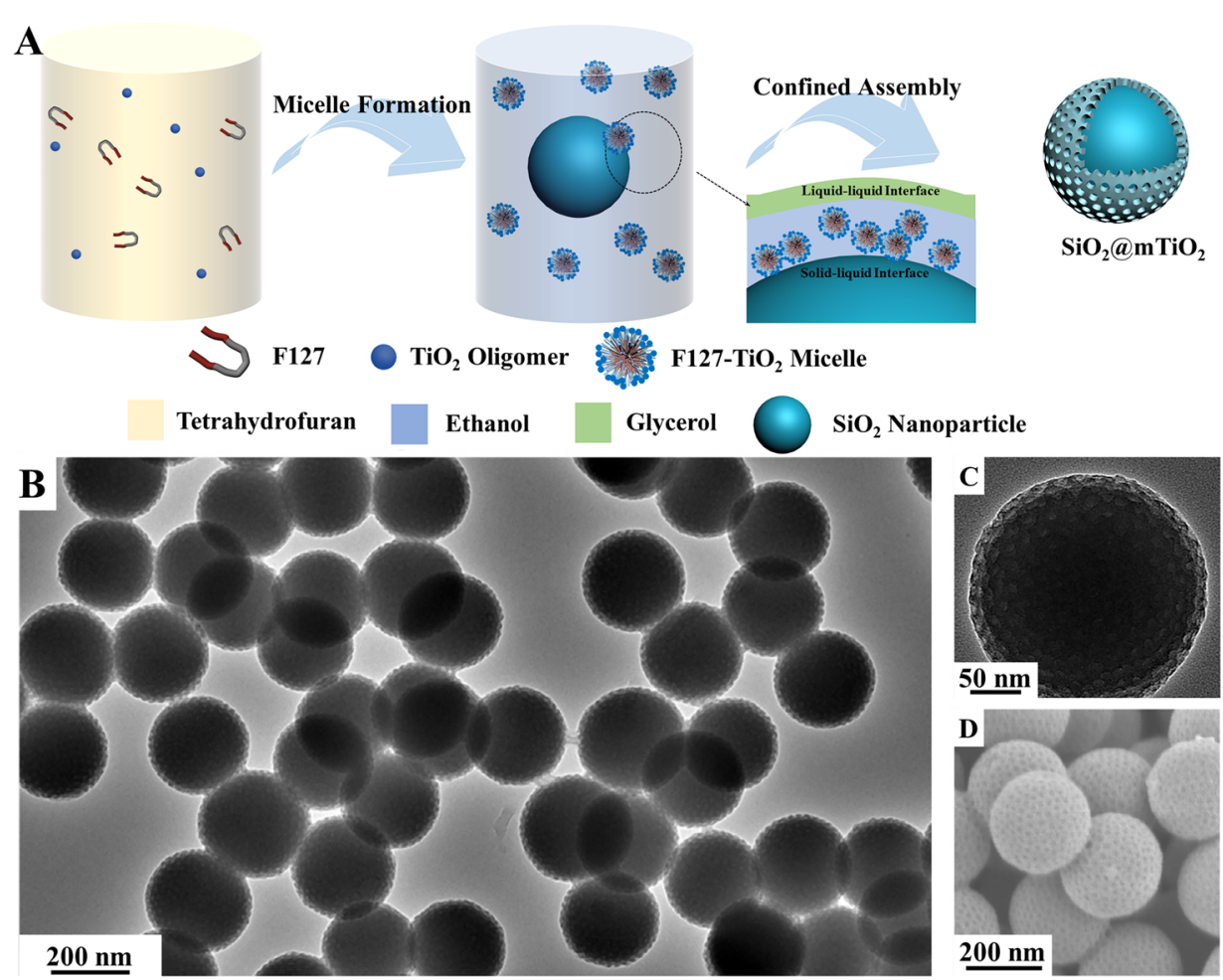

Figure 5. Manipulation of the solid-liquid-liquid interfaces for the single-layer core@shell structure. (A) Schematic illustrations and (B, C) TEM and (D) SEM images of the single-layered silica@ $\mathrm{mTiO}_{2}$ mesoporous nanoparticles fabricated through the solid-liquid-liquid triphase interfacial assembly. Reproduced with permission from ref 38. Copyright 2019 Cell Press.

the process stops. In order for micelle-precursor composites to assembly on the interface, their concentration must be higher than the critical concentration for precursor's heterogeneous nucleation on the interface, yet at the same time lower than its homogeneous self-nucleation concentration. Manipulation of this kinetic mediated nucleation and assembly process is the key in micelles' interfacial assembly for unique architectures.

\subsection{Manipulation of Solid-Solid Interfaces}

Solid-solid interfaces can be in situ formed during the micelle assembly, between the newly formed solid phase and the initial solid phase, which is further used to generate mesoporous nanomaterials with unique architectures. Utilizing the difference in hydrolysis rate between TEOS and organosilanes, the solid-solid interfaces are generated between the inner lowcross-linking degree silica and outer high-cross-linking degree organosilica in a $\mathrm{PMO}$ nanoparticles $(\mathrm{PMO}=$ periodic mesoporous organosilica). ${ }^{17}$ The hollow mesoporous structures can be obtained by selectively etching of the inner lowcross-linking silica. By repeatedly adding a mixture of TEOS and organosilane into the reaction system, multiple numbers of such low-cross-linking-high-cross-linking solid-solid interfaces can be formed in one nanoparticle. ${ }^{17}$ Thus, multilevel yolk@shell nanostructures are successfully obtained, the first 

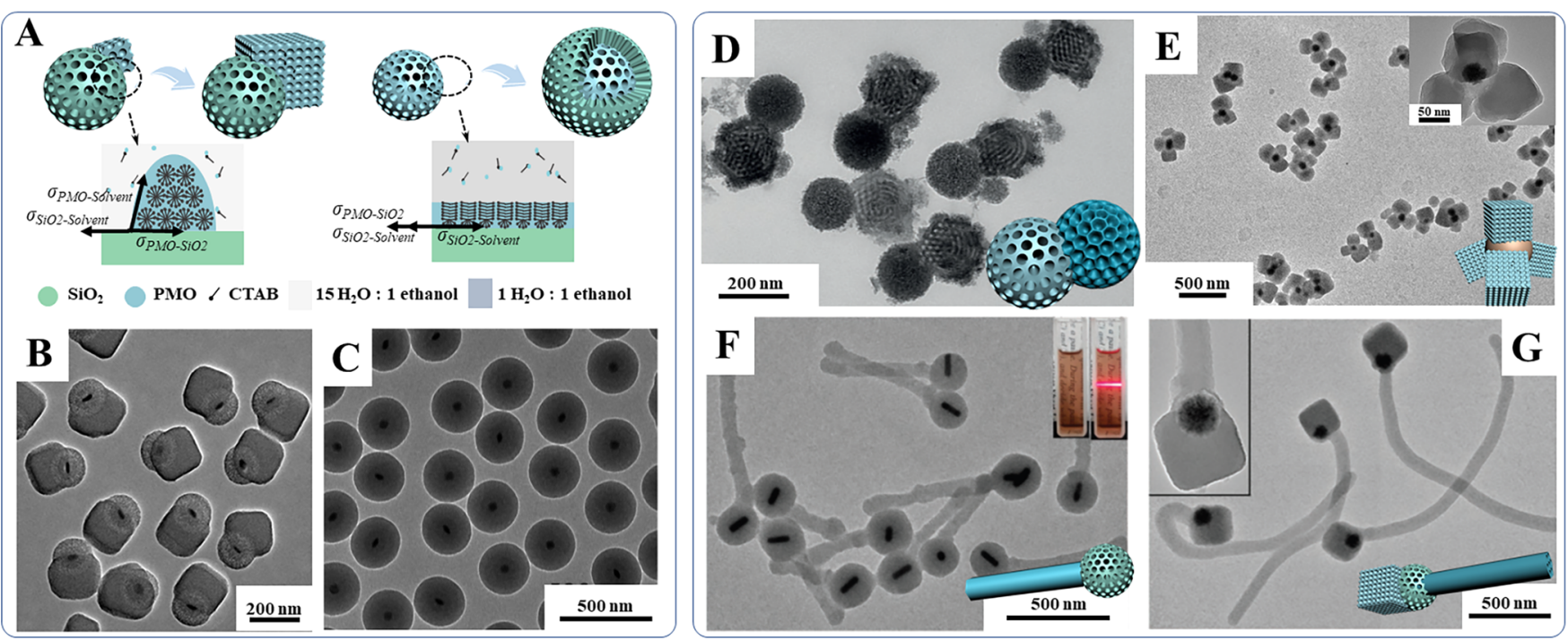

Figure 6. Asymmetric mesoporous nanoparticles obtained through interfacial energy mediated micelles' anisotropic assembly. (A) Schematic illustration of the interfacial energy meditated anisotropic assembly. TEM images of (B) Janus $\mathrm{UCNP} @ \mathrm{mSiO}_{2} \& \mathrm{PMO}(\mathrm{UCNP}=$ upconversion nanoparticle $=\mathrm{NaGdF}_{4}: \mathrm{Yb}, \mathrm{Tm} @ \mathrm{NaGdF}_{4}$ ) nanoparticles and $(\mathrm{C})$ symmetric core@shell structured $\mathrm{UCNP} @ \mathrm{mSiO}_{2} @ \mathrm{PMO}$ nanoparticles. (D) (G) TEM images of other asymmetric mesoporous architectures synthesized through interface energy induced anisotropic assembly: (D) dumbbell, (E) multipods, (F) asymmetric diblock, and (G) triblock one-dimensional nanorod architectures. Reproduced with permission from refs 18, 19, 22, and 41. Copyright 2014 American Chemical Society, 2019 Nature Publishing Group, 2017 John Wiley and Sons.

demonstration of achieving such architecture through an onepot fashion.

Normally, interfaces, especially solid interfaces are stable during reaction. On the basis of the swelling and shrinkage of $\mathrm{RF}$ upon soaking in or removal of $n$-hexane, the mesoporous $\mathrm{Fe}_{3} \mathrm{O}_{4} @ \mathrm{RF} @$ void@mSiO 2 yolk@shell structured nanoparticles were synthesized in a one-step fashion by Yue et al. (Figure 4A) ${ }^{37}$ In this experiment, the TEOS/hexane mixture is added into a solution containing $\mathrm{Fe}_{3} \mathrm{O}_{4} @ \mathrm{RF}$ nanoparticles, cationic surfactant $\mathrm{CTAB}$ and ammonia. The RF layer swells after contacting with hexane; thus, $\mathrm{CTAB} /$ silica-oligomer micelles are assembled on the swelled solid interface with an enlarged diameter. After removal of hexane solvent, RF shrinks back to the original size, leaving a void and forming a yolk@shell structure (Figure 4B-D). Such a one-step approach to prepare yolk@shell structure is a very exquisite utilization of solidsolid interface for the micelle assembly.

Besides interfaces formed in two-phase systems (liquidliquid, solid-liquid, solid-solid), the combined effect of three phases brings further complex systems. For example, the solid-liquid-liquid interface is designed by introducing the solid silica nanospheres into the above-mentioned ethanolglycerol liquid-liquid system (section 2.1). The Pluronic $\mathrm{F} 127 / \mathrm{TiO}_{2}$-oligomer micelles can be confined in the ethanol interlayer between the solid nanoparticles and high viscosity glycerol layer (Figure 5A). Thus, precisely controlled coating of single-layered mesoporous $\mathrm{TiO}_{2}$ on colloidal $\mathrm{SiO}_{2}$ nanoparticles is achieved through this solid-liquid-liquid triplephase interfacial assembly (Figure $5 \mathrm{~B}-\mathrm{D}) .{ }^{38}$ By tuning the amount of glycerol, the number of mesoporous $\mathrm{TiO}_{2}$ layers coated on the colloidal silica nanospheres can be precisely controlled from one to five. This approach is extremely universal and can be used for the coating of mesoporous $\mathrm{TiO}_{2}$ layers on various nanomaterials, such as carbon nanotubes, graphene oxides, $\mathrm{Fe}_{2} \mathrm{O}_{3}$ ellipsoids, etc.

\section{INTERFACIAL-ASSEMBLY-DIRECTED ASYMMETRIC MESOPOROUS NANOMATERIALS}

Due to the asymmetries in geometry, chemical composition, surface property, and functionality, asymmetric nanomaterials have aroused great attention recently. However, present asymmetric nanomaterials are mainly nanocrystals or polymers, with a low surface area and lacking functionalities. ${ }^{39}$ Recently, the interface directed anisotropic assembly of micelles has paved a way for fabricating asymmetric mesoporous nanomaterials. When the micelles are uniformly spread or isotropically assembled on interfaces, the obtained mesoporous nanomaterials show uniform core@shell structures. Isotropical assemble for symmetric architecture is usually energy favored. However, when an energy is provided so that they are directed into an anisotropic manner on the interface, unique asymmetric mesoporous nanomaterials can be obtained. These asymmetric mesoporous nanomaterials with independent mesoporous compartments, tunable spatial isolated surfaces and compositions, have great application potentials in various fields. Our group has done a lot of works in this field, obtaining a series of mesoporous nanomaterials with unique asymmetric architectures.

\subsection{Interfacial Energy Mediated Island Growth for Asymmetric Mesoporous Nanostructures}

In 2013, Li et al. first demonstrated the anisotropic growth of mesoporous materials based on interfacial energy induced island growth of mesopore channels (Figure 6A). ${ }^{18}$ In this work, the obtained Janus mesoporous nanoparticles consisted of one spherical mesoporous silica $\left(\mathrm{mSiO}_{2}\right)$ compartment with radial mesopore channels and a cubic PMO compartment with $P m \overline{3} n$ mesostructure. Such unique mesoporous heterodimers with precisely controlled two-compartment correspondence is the first of its kind. The Janus architecture is achieved through anisotropic growth of $\mathrm{PMO}$ on $\mathrm{mSiO}_{2}$ surface. The solid-solid interfacial energy between $\mathrm{mSiO}_{2}$ and $\mathrm{PMO}$ plays a critical role in the interfacial-assembly process, the change in total surface 

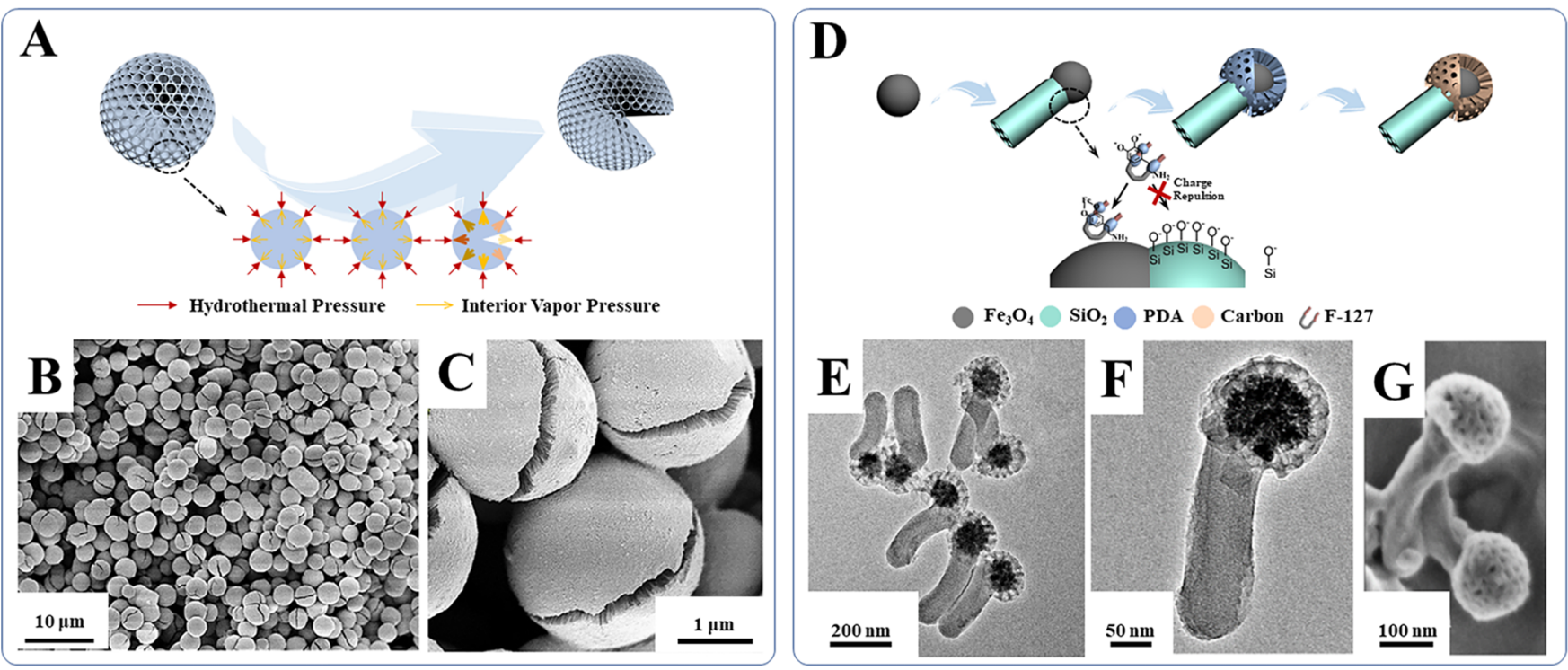

Figure 7. Asymmetric mesoporous nanoparticles obtained through internal vapor pressure and selective encapsulation. Schematic illustrations, TEM and SEM images of asymmetric mesoporous nanoparticles synthesized through various methods: (A) $-(\mathrm{C})$ mesoporous $^{\mathrm{TiO}} \mathrm{O}_{2}$ nanoparticles with controllable asymmetric cracking synthesized through internal vapor pressure and $(\mathrm{D})-(\mathrm{G})$ dual-mesoporous asymmetric $\mathrm{Fe}_{3} \mathrm{O}_{4} @$ $\mathrm{mPDA} \& \mathrm{mSiO}_{2}$ nanoparticles synthesized through charge-induced selective encapsulation. Reproduced with permission from refs 43 and 44. Copyright 2018 Cell Press and 2018 American Chemical Society.

energy can be expresses as $\Delta \sigma=\sigma_{\mathrm{PMO}-\text { solvent }}+\sigma_{{\mathrm{PMO}-\mathrm{SiO}_{2}}_{2}}$ $\sigma_{\mathrm{SiO}_{2} \text {-solvent }}$. At a high water-ethanol ratio, relative hydrophilic environment makes the $\sigma$ between hydrophobic PMO and solvent very large, so that $\Delta \sigma>0$. Thus, the interfacial energy forbids the hydrophobic PMO to spread on the surface of $\mathrm{mSiO}_{2}$; thus, PMOs tend to island grow on the silica surface, leading to asymmetric $\mathrm{mSiO}_{2} \& \mathrm{PMO}$ multicompartment nanoparticles (Figure 6B). At a low water-ethanol ratio, relative hydrophobic environment favors the growth of PMOs to spread on the silica surface, resulting in uniform coating and core@shell structures (Figure 6C). In a following work, they allowed the anisotropic growth of the PMOs to be continued. The $\mathrm{mSiO}_{2}$ nanoparticles are encapsulated by PMO spheres but not located at the center of the entire architecture, resulting in an eccentric core@shell structure. ${ }^{40}$ After etching the silica part, asymmetric single-hole nanocages are successfully obtained. This asymmetric structure is made of one eccentric void with opening and a mesoporous shell. Different from the conventional hollow structure, the guests loaded in the large vacancy can enter and exit the hollow through the opening, rather than having to pass through the shells.

This interfacial energy mediated anisotropic growth of mesoporous compartments is universal, which can be easily generalized to other initial compartments with different properties, enabling a series of following works, including the anisotropic growth of $\mathrm{mSiO}_{2}$ and $\mathrm{PMOs}$ on metals, ${ }^{41}$ polymers (Figure 6D), ${ }^{22}$ etc. Besides single-site nucleation that led to heterodimer structures, Zhao et al. controlled the number of anisotropic nucleation site through tuning surface kinetics and obtained a series of mesoporous multipods nanocomposites with controllable surface architectures (Figure 6E). ${ }^{19}$ Taking the anisotropic assembly of $\mathrm{CTAB} /$ silica-oligomer micelles on $\mathrm{Fe}_{3} \mathrm{O}_{4} @ \mathrm{SiO}_{2} @ \mathrm{RF}$ nanoparticles as an example, different from the $\mathrm{SiO}_{2}$ initial surface for single-site anisotropic assembly, the RF surface has abundant phenolic hydroxyl groups with strong nucleophilicity. Therefore, the reaction activity of silane nucleophilic substitution (i.e., nucleation capability) on the RF surface is higher than that on the $\mathrm{SiO}_{2}$ surface, rendering the RF surface a lower critical nucleation concentration of surfactants/silicate oligomers. Nucleation of silanes thus starts earlier on the RF surface, leading to higher speed of silane oligomer generation at the nucleation start, long nucleation duration time, and thus continuous multiple nucleation. What is more, the number of nucleation sites can be precisely controlled from one (Janus nanostructure) to four (tetrapods nanostructure) by mediation of the surface properties of RF. This surface-kinetics mediated multisite nucleation strategy provides a pathway in the rational design of functional mesoporous nanoparticles with controllable surface topological structures.

Besides the anisotropic assembly of cubic (space group $P m \overline{3} n$ ) mesostructured nanocubes, the methodology can also be generalized to hexagonal PMO nanorods. Li et al. developed a series of functional asymmetric 1D mesoporous nanoparticles, which consisted of the closely connected spherical compartment and rod-like compartment (Figure 6F). ${ }^{41}$ The formation mechanism of the asymmetric nanorods is similar to the anisotropic growth of cubic PMO nanoparticles mentioned previously, only that $\mathrm{CTAB} /$ silane-oligomer micelles are assembled as hexagonal manner to form mesostructured nanorods. Combining the anisotropic assembly of cubic and hexagonal mesostructures, exquisite triblock asymmetric mesoporous nanoparticles containing three domains of sphere, cube, and rod can be fabricated (Figure 6G), which further extended the range of multicompartment mesoporous nanoparticles.

On the basis of the interfacial energy induced anisotropicassembly strategy, Wang et al. anisotropically assembled PMO nanorods on the presynthesized virus-like mesoporous silica nanoparticles with a rough surface, forming a "nanotruck" architecture with a rough virus-like head and a smooth PMO tail. $^{42}$ The authors have systematically investigated the influence of the reaction parameters on the anisotropic 
assembly of mesoporous domain on the rough surface. They found that isotropic and anisotropic assembly of PMO always coexisted, inducing the loss of the surface functionality of the virus-like nanoparticles. The high alkalinity leads to the domination of the anisotropic growth, which further results in the preservation of the rough surface. These data would provide vital information for future researchers.

\subsection{Other Approaches for the Fabrication of Asymmetric Mesoporous Nanostructures}

Besides interfacial energy mediated island growth strategies, there are numerous other methods for the anisotropic assembly of micelles. For example, internal evaporation force induced anisotropic assembly, ${ }^{43}$ selective growth based on varied surface chemistry, ${ }^{44}$ etc.

Nanoparticles may generate internal pressure during reaction, which is fine controlled and which would prove useful for architecting asymmetric nanostructures. In a typical example, mesoporous $\mathrm{TiO}_{2}$ microspheres are first synthesized on the basis of the homogeneous assembly. Then through carful manipulation of external hydrothermal pressure as well as internal vapor pressure of the microspheres during the further hydrothermal treatment, mesoporous $\mathrm{TiO}_{2}$ nanoparticles with controllable asymmetric cracking are obtained (Figure 7A). ${ }^{43}$ During this hydrothermal process for the further condensation of the $\mathrm{TiO}_{2}$ framework, the butanol generated from the hydrolysis of tetrabutyl titanate as well as water and THF that remain inside the microspheres evaporate, resulting in increased internal vapor pressure. The interior vapor pressure increases continuously during the reaction process, which in the end exceeds the exterior hydrothermal pressure. Then, the microspheres are suddenly burst, forming $\sim 800 \mathrm{~nm}$ mesoporous $\mathrm{TiO}_{2}$ nanospheres with controlled openings (Figure 7B,C). Fang et al. also took advantage of the confinement effect and inner tension induced shrinkage, obtaining asymmetric mesoporous carbon hemispheres. ${ }^{20}$ Mesoporous polymer@mSiO $\mathrm{m}_{2}$ core@shell structures are first formed and then calcinated to carbonize the inner polymer. Polymer shrinks during calcination, yet at the same time the interface tension between polymer-silica interface coexists. The contraction of the polymer goes along a particular polar direction to retain the connection with the silica shell, in the end leading to asymmetric mesoporous hemispheres. These asymmetric mesoporous nanomaterials obtained on the basis of pressures demonstrate a novel way of architecting anisotropy in nanomaterials.

Selective etching is another method of architecting asymmetric structures. In a typical example, PMO nanospheres are first synthesized by assembling TEOS and organosilane together with cationic surfactant CTAB. ${ }^{45}$ Then the selective etching process is carried out on the basis of the difference in cross-linking degree between inside (mainly low-cross-linked silica) and outside (mainly high-cross-linked PMO) of the nanospheres. Not only the interiors but also parts of the outer layers are etched, leaving a "soft" nanocapsules with thin shell as well as flexible $\mathrm{Si}-\mathrm{R}-\mathrm{Si}$ organosilica framework. The upper half of the nanocapsules shrinks and deforms to the low half to form an asymmetric bowl-like morphology.

Wang et al. used interface-directed coassembly in confined space to synthesize mesoporous silica hemispheres. ${ }^{34}$ In their work, ethanol solution of P123 and TEOS is evaporated with the existence of $3 \mathrm{D}$ ordered macroporous carbon (3DOMC). During evaporation of ethanol, silane-surfactant precursor solution can enter the macropores of the 3DOMC due to capillary force. Precursors are concentrated at the bottom of the macropores due to gravity, and in the end uniform mesostructured silica hemispheres are formed within the macropores. Burning out the 3DOMC in air results in asymmetric mesoporous silica hemispheres. What's more, instead of $\mathrm{SiO}_{2}$ nanospheres with $\mathrm{Fe}_{3} \mathrm{O}_{4} @ \mathrm{SiO}_{2}$ nanospheres to form the 3DOMC, one $\mathrm{Fe}_{3} \mathrm{O}_{4}$ nanosphere can be introduced into each macropore. The $\mathrm{Fe}_{3} \mathrm{O}_{4}$ nanospheres are unable to be retained at the center of the macropores due to lack of support. After impregnation of silane precursors, eccentric core@shell structured $\mathrm{Fe}_{3} \mathrm{O}_{4} @ \mathrm{mSiO}_{2}$ nanoparticles are obtained.

Premade asymmetric nanoparticles can also be used as templates to induce new asymmetric mesoporous nanoparticles, as varied compartments of asymmetric nanoparticles provide different interfaces. Zhao et al. utilized the surfacecharge induced selective encapsulation of mPDA (mesoporous polydopamine) on asymmetric $\mathrm{Fe}_{3} \mathrm{O}_{4} \& \mathrm{mSiO}_{2}$ nanoparticles, obtaining dual-mesoporous asymmetric $\mathrm{Fe}_{3} \mathrm{O}_{4} @ \mathrm{mPDA} \& \mathrm{~m}-$ $\mathrm{SiO}_{2}$ nanoparticles (Figure 7D). ${ }^{44}$ The multicompartment mesoporous structure is made up of one $\mathrm{Fe}_{3} \mathrm{O}_{4} @ \mathrm{mPDA}$ nanosphere at the end of a $\mathrm{mSiO}_{2}$ nanorod, and further calcination led to asymmetric $\mathrm{Fe}_{3} \mathrm{O}_{4} @ \mathrm{mC}_{\mathrm{m}} \mathrm{mSiO} \mathrm{O}_{2}$ nanoparticles (Figure 7E,G). The selective encapsulation is induced by the different surface chemistry between $\mathrm{SiO}_{2}$ and $\mathrm{Fe}_{3} \mathrm{O}_{4}$ : negative charged $\mathrm{SiO}_{2}$ interface repels negative charged PDA, while $\mathrm{Fe}^{3+}$ can coordinate with dopamine molecules to facilitate the interfacial assembly of F127/PDA-oligomer micelles. This work is the first report of spatial isolation of carbon and silica at single-particle level and provides potential in many fields.

\section{UNIQUE ARCHITECTURE ENHANCED APPLICATIONS}

These mesoporous nanomaterials with unique architectures in morphologies, composition and surface properties, are very useful for many applications. The interaction between mesoporous nanomaterials and surroundings (e.g., drug molecules, cell membrane, emulsion interface, etc.) is altered due to the unique architecture, therefore providing enhanced performances. In this section, we demonstrate a few typical examples for the applications of these mesoporous nanomaterials in the fields of catalysis, energy storage and conversion, biomedicine, etc.

\subsection{Enhanced Applications of Mesoporous Nanomaterials with Unique Symmetric Architectures}

Although the nanosphere is one of the most common symmetric shapes, the above-mentioned interfacial assembly strategies can endow the mesoporous nanospheres with brandnew mesostructures, interior composition, and surface structures, which are optimal settings for many applications.

First of all, mesoporous nanoparticles with a large pore size can facilitate immobilization of guests with large diameters. Yue et al. fabricated mesoporous $\mathrm{Fe}_{3} \mathrm{O}_{4} @ \mathrm{RF} @ \mathrm{mSiO}_{2}$ nanoparticles with large and tunable perpendicular mesopores $(5.0-9.0 \mathrm{~nm})$ through biphasic interfacial-assembly method. ${ }^{28}$ On the basis of the large and open mesopores, the mesoporous nanoparticles with a pore size of $\sim 9.0 \mathrm{~nm}$ are used to immobilize a large-diameter enzyme trypsin $(\sim 4.0 \mathrm{~nm})$ and exhibited an excellent size selective enzymolysis of low molecular proteins in the mixture of proteins with different 

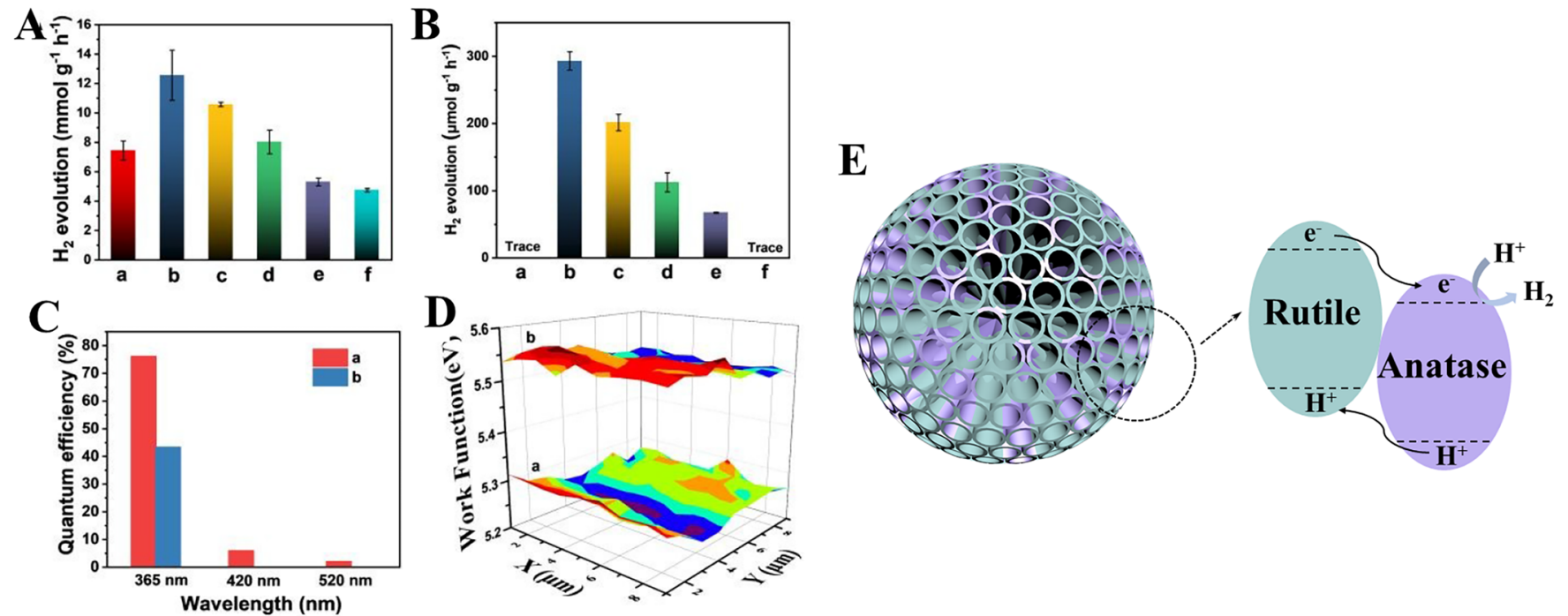

Figure 8. Junction induced mesoporous $\mathrm{TiO}_{2}$ nanoparticles with enhanced photocatalysis performance. $\mathrm{H}_{2}$ evolution rates of mesoporous $\mathrm{TiO} \mathrm{O}_{2}$ microspheres with various ratio of anatase/rutile (a) - (e) and commercial P25 (f) under (A) AM $1.5 \mathrm{G}$ and (B) visible light: (a) 100/0, (b) 77/23,

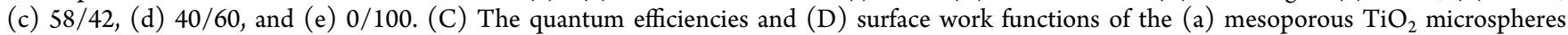
with an anatase/rutile ratio of $77 / 23$ and (b) commercial P25. (E) Schematic diagram illustrating the process of $\mathrm{H}_{2}$ evolution across the mesoporous $\mathrm{TiO}_{2}$ microspheres with abundant anatase-rutile junctions. Reproduced with permission from ref 46 . Copyright 2019 Royal Society of Chemistry.

sizes. Besides the enzyme, the mesoporous nanoparticles with large mesopores have also been utilized for loading of other biomacromolecule, such as RNA, protein, etc.

Mesoporous frameworks may provide stable substrate for the immobilization of catalytic active sites as well as facilitate mass transfer, which are both crucial in catalytic applications. Several works from our group have demonstrated that by immobilizing catalytic sites within the mesopore frameworks, both catalytic efficiency and the long-term stability can be significantly enhanced. ${ }^{23}$ Liu and Lan et al. immobilized $\mathrm{Au}$ nanocrystals on the bouquet-like mesoporous $\mathrm{TiO}_{2}$ superstructures. ${ }^{16}$ They demonstrated that the high architectural complexity, high accessible surface area, radially orientation, and high interconnection of the meso-channels of the superstructure may greatly enhance the catalytic performance. High surface area enables easier immobilization of catalytic $\mathrm{Au}$ nanocrystals, while the superstructure provides the nanoparticles increased interaction with surrounding solution, resulting in efficient mass transfer. With cis-semihydrogenation of alkynes as a model, both the activity and selectivity of the nanocatalysts increase with the increasing of architecture complexity. This is attributed to more reaction spaces being provided in the superstructured mesoporous $\mathrm{TiO}_{2}$, and therefore higher activity and selectivity are achieved for semihydrogenation reactions.

High crystallization is also crucial in photo- and electrocatalysis. Architecting crystallized mesoporous semiconductors has been attracting considerable attention because of their large accessible active surfaces. The mesoporous $\mathrm{TiO}_{2}$ microspheres fabricated by Zhang et al. with abundant anatase-rutile junctions exhibited excellent performance in photoelectrochemical conversion and photocatalytic water splitting (Figure $8 \mathrm{~A}-\mathrm{E}){ }^{46}$ The neatly controlled anataserutile junctions as well as high surface area endow the nanomaterials with $\mathrm{H}_{2}$ evolution rate of as high as $293 \mu \mathrm{mol}$ $\mathrm{g}^{-1} \mathrm{~h}^{-1}$ under the light of $\lambda>400 \mathrm{~nm}$, which is much higher than that of the commercial P25. The present fabricated mesoporous $\mathrm{TiO}_{2}$ microspheres are "highly crystallized"; it would be much favored if "single crystallized" mesoporous semiconductor nanomaterials can be obtained, as such architecture may significantly facilitate electron transfer and enhance performances.

The nanomaterials' interactions with biological interfaces, which are significantly affected by the nanoparticles' surface physicochemical properties (roughness, stiffness, etc.), are crucial for intracellular drug delivery, bacterial inhibition, and so on. Therefore, mesoporous nanomaterials with unique architectures may provide novel interaction behaviors with biological interfaces. For example, Wang et al. have fabricated virus-like mesoporous silica nanoparticles with a spiky tubular rough surface. ${ }^{15}$ This unique biomimetic morphology endows the virus-like nanoparticles enhanced interaction with cell membrane and increased cellular uptake comparing to mesoporous silica nanospheres with smooth surface. A small disadvantage of this architecture is that, as the surface of the virus-like nanoparticles are covered with spicks, they suffer from relative low surface area. Though this can be conquered by post hydrothermal treatment, this shortcoming still hinders applications such as drug delivery. In a similar fashion, Zhao et al. also utilized the multivalent-interactions enhanced nanobio interactions from mesoporous $\mathrm{Fe}_{3} \mathrm{O}_{4} @ \mathrm{SiO}_{2} @ \mathrm{RF} \& \mathrm{PMO}$ multipods to achieve enhanced bacterial adhesion and inhibition. ${ }^{19}$

Besides the roughness, the surface stiffness of the nanoparticles can also influence the endocytosis. Teng et al. obtained hollow PMO nanocapsules through a preferential etching approach, which was based on the interface between low-cross-linked and high-cross-linked silica. ${ }^{45}$ As the entire interior and part of the shell are etched, the hollow mesoporous nanocapsules are "soft" and deformable. During cellular internalization, the change of morphology (i.e., deformability) reduces the deformation of the cellular membranes and the corresponding energy during internalization, enabling higher cellular uptake comparing to "hard" control groups (Figure 9A-D). 
A
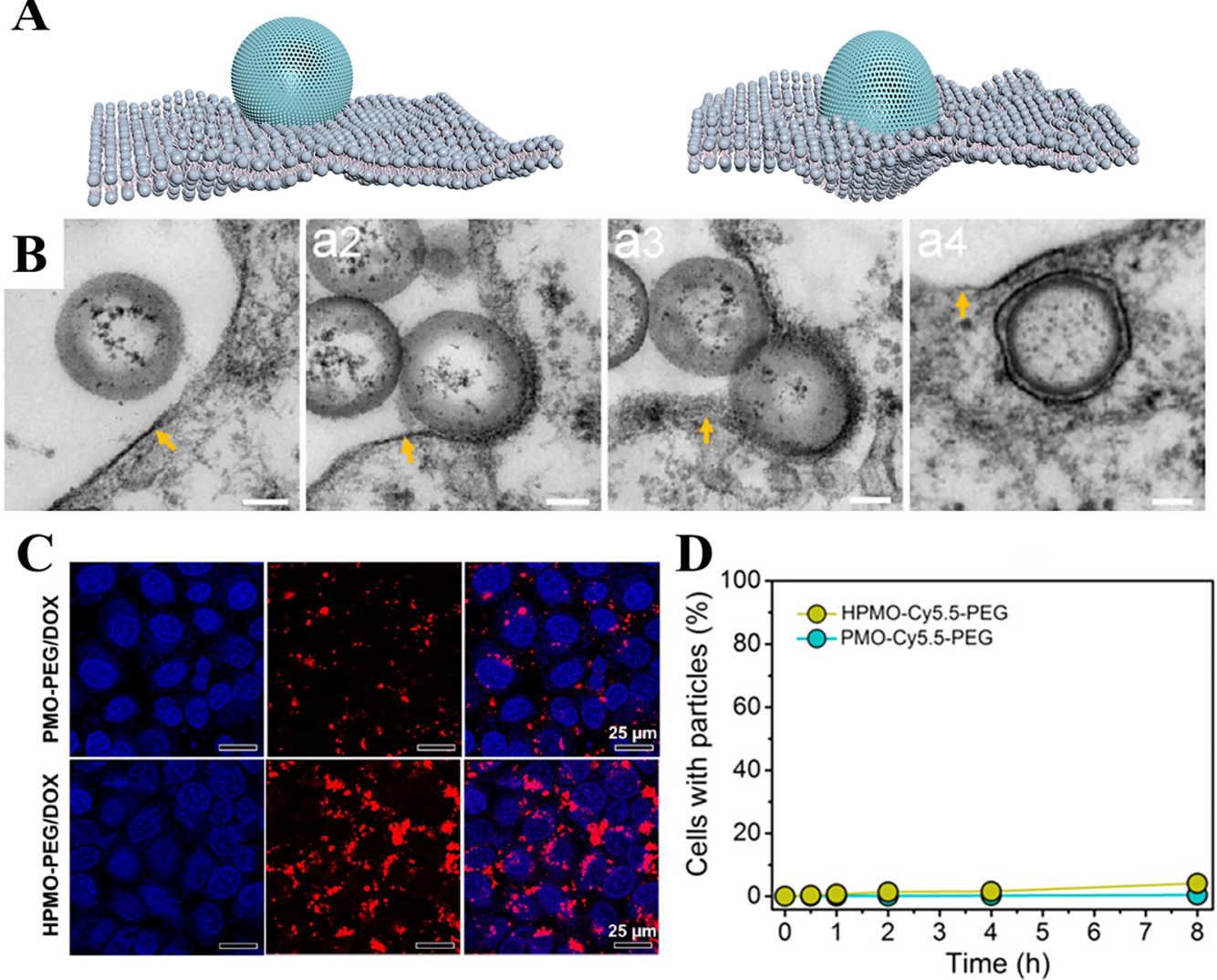

Figure 9. Enhanced cellular internalization of deformable mesoporous nanoparticles. (A) Schematic illustration and (B) TEM images of the deform-assisted uptake processes of deformable hollow PMO nanocapsules by human breast cancer MCF-7. (C) Fluorescent confocal micrographs and (D) cellular uptake measured by flow cytometry of MCF-7 human breast cancer cells incubated with $100 \mu \mathrm{g} \mathrm{mL} \mathrm{L}^{-1}$ of (up) deformable hollow PMO nanocapsules and (bottom) nondeformable hollow PMO nanospheres. Reproduced with permission from ref 45 . Copyright 2018 American Chemical Society.

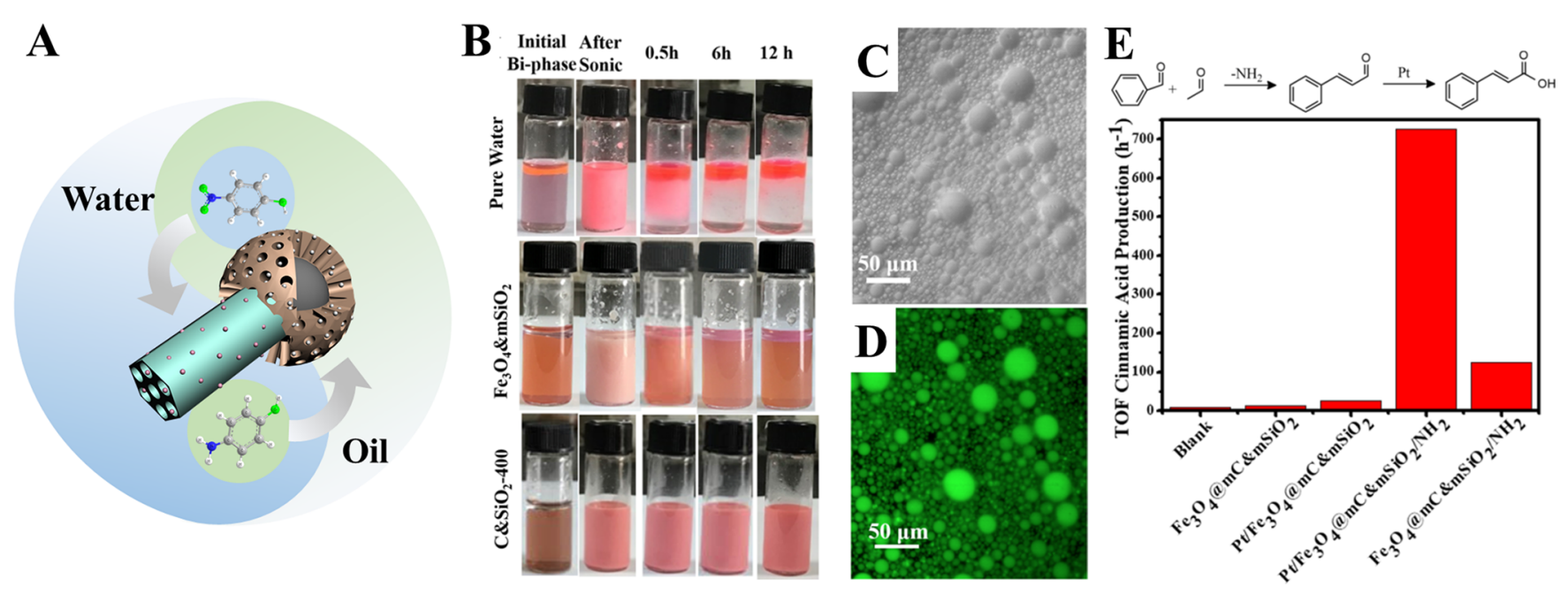

Figure 10. Emulsion-catalysis of the asymmetric mesoporous nanocomposites. (A) Schematic illustration of asymmetric mesoporous $\mathrm{Fe}_{3} \mathrm{O}_{4} @$ $\mathrm{mC} \& \mathrm{mSiO}_{2}$ nanoparticles. (B) Optical micrograph image and (C, D) fluorescent optical micrograph image of the toluene-in-water emulsion stabilized by pure water, the asymmetric mesoporous $\mathrm{Fe}_{3} \mathrm{O}_{4} \& \mathrm{mSiO}$ nanoparticles, and mesoporous $\mathrm{Fe}_{3} \mathrm{O}_{4} @ \mathrm{mC} \& \mathrm{mSiO} \mathrm{O}_{2}$ nanoparticles with spatial carbon and silica isolation. (E) Schematic illustration of the catalytic cascade reaction over the asymmetric $\mathrm{Pt}_{2} / \mathrm{Fe}_{3} \mathrm{O}_{4} @ \mathrm{mC}_{2} \mathrm{mSiO} \mathrm{O}_{2} / \mathrm{NH}_{2}$ mesoporous nanoparticles stabilized biphasic catalysis system and Cinnamic acid's production turnover frequency of the cascade reaction using different functionalized nanoparticles as catalysts: the asymmetric mesoporous $\mathrm{Fe}_{3} \mathrm{O}_{4} @ \mathrm{mC}_{2} \mathrm{mSiO}_{2}$ nanoparticles without functionalization $\left(\mathrm{Fe}_{3} \mathrm{O}_{4} @ \mathrm{mC} \& \mathrm{mSiO}_{2}\right)$, with only $\mathrm{Pt}$ nanocrystals loaded in the carbon domain $\left(\mathrm{Pt} / \mathrm{Fe}_{3} \mathrm{O}_{4} @ \mathrm{mC}_{\mathrm{m}} \mathrm{mSiO}_{2}\right)$, with both Pt nanocrystals loaded in the mesoporous carbon domain and $-\mathrm{NH}_{2}$ groups functioning in the mesoporous $\mathrm{SiO}_{2}$ domain $\left(\mathrm{Pt} / \mathrm{Fe}_{3} \mathrm{O}_{4} @ \mathrm{mC} \& \mathrm{mSiO}_{2} / \mathrm{NH}_{2}\right)$, and with only $-\mathrm{NH}_{2}$ groups functioning in the $\mathrm{SiO}_{2}$ domain $\left(\mathrm{Fe}_{3} \mathrm{O}_{4} @ \mathrm{mC} \& \mathrm{mSiO} \mathrm{O}_{2} / \mathrm{NH}_{2}\right)$. Reproduced with permission from ref 44. Copyright 2018 American Chemical Society. 
A

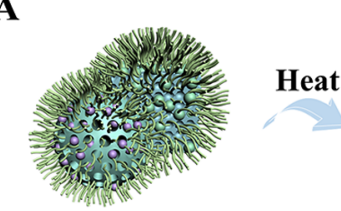

Hydrophilic Drug

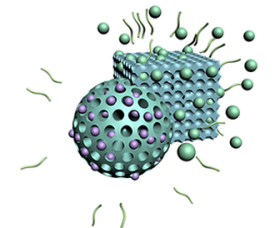

Hydrophobic Drug

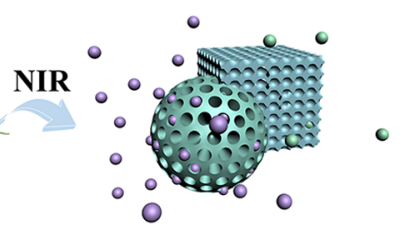

Thermal-responsive Polymer
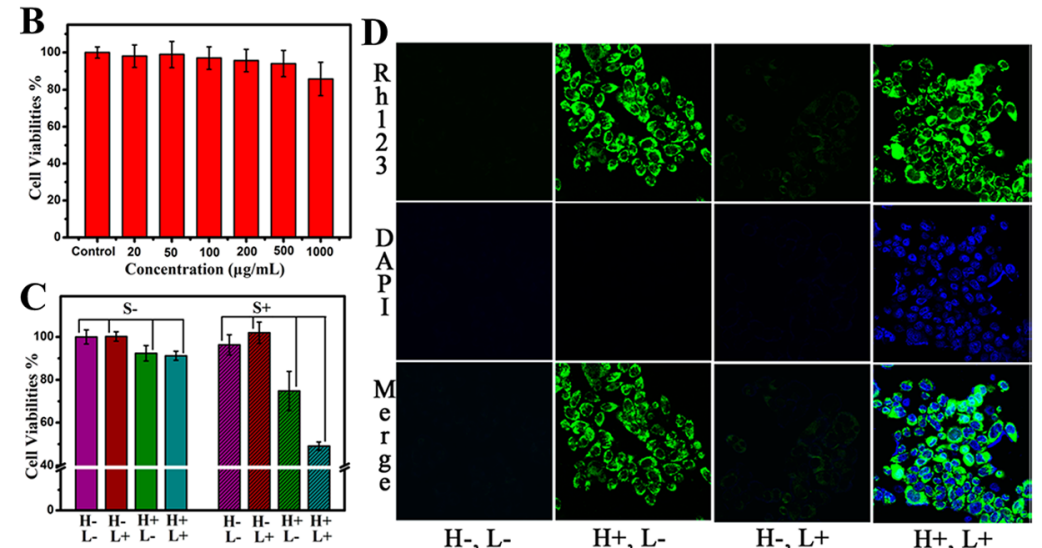

H-, L-

$\mathrm{H}+, \mathrm{L}-$

$\mathrm{H}+, \mathrm{L}+$

Figure 11. Dual-model drug release of the asymmetric mesoporous nanocomposites. (A) Schematic presentation for dual-control drug release from dual-compartment UCNP@SiO${ }_{2} @ \mathrm{mSiO}_{2} \& \mathrm{PMO}\left(\mathrm{UCNP}=\right.$ upconversion nanoparticle $=\mathrm{NaGdF}_{4}: \mathrm{Yb}_{\mathrm{Tm}} @ \mathrm{TaGdF}_{4}$ ) nanoparticles. (B) Cell viability assay of Janus nanocomposites on HeLa cells. (C) Cell viabilities of paclitaxel and DOX coloaded Janus nanocomposites under the heat (H) and NIR light (L) treatment (S means sample). (D) Confocal laser scanning microscopy (CLSM) observations of the HeLa cells after incubation with the Rh123 (green) and DAPI (blue) coloaded mesoporous Janus nanocomposites with or without heat (H) and NIR light (L) stimuli. Reproduced with permission from ref 18. Copyright 2014 American Chemical Society.

\subsection{Enhanced Applications of Mesoporous Nanomaterials with Unique Asymmetric Architectures}

Compared with the symmetric mesoprous nanomaterials, the asymmetric nanocomposites are ideal for multiple-component conjugations, loading, or designing of smart delivery system at the single-particle level. Most importantly, each mesoporous component can interact with surrounding environments independently and in various manners, which is based on size, charge, hydrophilicity, etc., showing great potential for biomedicine, catalysis and so on.

In the catalysis field, due to the fact that many frequently used reaction substrates are not water-soluble, the water-oil emulsion is often applied for biphasic catalysis. Yet emulsions are usually instable, and the interaction area between water-oil is often low. Stabilization of emulsion for maximum water-oil interface area is required, and such demand can be met by asymmetric nanoparticles with distinct hydrophilic and hydrophobic domains. Such asymmetric mesoporous nanoparticles can load catalytic sites to assist the reaction, as well as function as "surfactants" to stabilize the emulsions, thus enhancing biphasic catalysis reaction efficiencies.

Yang et al. utilized the anisotropic assembly of PMO on $\mathrm{Pt}$ nanocrystals loaded RF nanoparticles to obtain dumbbell shaped multicompartment Pt/RF\&PMO nanoparticles. The $\mathrm{Pt} / \mathrm{C} \& \mathrm{PMO}$ catalyst can be obtained after carbonization. ${ }^{22}$ As one compartment of the particle is hydrophobic carbon and the other side was hydrophilic PMO, the hydrophilicity variation endows the Janus nanoparticle excellent Pickering emulsion stabilization ability and thus enhanced emulsion catalytic performance. Yet in this system, due to the usage of PMOs, which is naturally a mixture of carbon and silica, the adjusting of hydrophilicity is limited. Zhao et al. synthesized asymmetric mesoporous $\mathrm{Fe}_{3} \mathrm{O}_{4} @ \mathrm{mC} \mathrm{m} \mathrm{miO}_{2}$ nanoparticles, which for the first time have single-particle level carbon/silica spatial isolation (Figure 10A). ${ }^{44}$ Due to such isolation, the size ratio of hydrophilic/hydrophobic domain can be precisely tuned. These nanoparticles function as "solid surfactants" with tunable emulsion stabilization ability (Figure 10B-D). They also selectively functionalized $\mathrm{Pt}$ nanocrystals and amino groups on carbon and silica compartment of the Janus nanoparticles. Based on such asymmetric modification, a biphasic cascade reaction that transfers benzaldehyde and acetaldehyde to cinnamaldehyde and finally to cinnamic acid in a one-pot fashion is achieved (Figure 10E).

To realize the best therapeutic effect, the species and doses of drugs should be optimized at different clinical manifestations and periods in the treatment. In asymmetric mesoporous nanostructures, on the basis of the fact that different compartments have varied properties, such as chemical property, pore size, etc., each compartment can be controlled to load only one kind of guests. Then these guests can be released independently by designing different triggers on each compartment of the asymmetric mesoporous nanoparticles, realizing optimized therapeutic effects.

$\mathrm{Li}$ et al. have synthesized multicompartment asymmetric UCNP@SiO $2 @ \mathrm{mSiO}_{2} \& \mathrm{PMO}$ (UCNP = upconversion nanoparticle $\left.=\mathrm{NaGdF}_{4}: \mathrm{Yb}, \mathrm{Tm} @ \mathrm{NaGdF}_{4}\right)$ nanoparticles. The asymmetric mesoporous nanoparticles possess two separated compartments with different hydrophilicity: a hydrophilic $\mathrm{mSiO}_{2}$ and a hydrophobic PMO. As shown in Figure $11 \mathrm{~A},{ }^{18}$ hydrophilic DOX molecules are encapsulated in the UCNP@ $\mathrm{mSiO}_{2}$ compartment and blocked by photosensitive azobenzene (Azo) switch, and the hydrophobic drug paclitaxel is loaded in PMO. After further coating the multicompartment nanoparticles with heat sensitive phase change material of 1tetradecanol, the two guests can be independently released 
upon heat (which cleavage 1-tetradecanol) and NIR light (transferred by UCNP into ultraviolet to trigger Azo switches, Figure $11 \mathrm{~B}-\mathrm{D})$. This is the first successful attempt of dualdrugs' sequential release. The protocol of amphiphilicity induced multidrug release through asymmetric multicompartment mesoporous nanoparticles is inherited by several other works.

The size of loaded guests also varies from molecule level to several nanometers. Therefore, the size effect can also be used for the designing of multiguests coloading and controllable release. $\mathrm{Li}$ et al. utilized the eccentric single-hole hollow mesoporous nanoparticles to load small molecule drugs and large sized proteins. ${ }^{40}$ In this unique single-hole nanocages, the hollow cavity and big hole $(\sim 25 \mathrm{~nm})$ can serve as a storage space and passage for large sized albumin from bovine serum (BSA, $\left.2.1 \times 4 \times 1.4 \mathrm{~nm}^{3}\right)$. Meanwhile, the mesopores $(2-10$ $\mathrm{nm})$ with a high surface area $\left(\sim 500 \mathrm{~m}^{2} \mathrm{~g}^{-1}\right)$ in the silica shells can provide storage space for DOX molecules $\left(<1 \mathrm{~nm}^{3}\right)$, therefore realizing the independent loading and release of two different guests based on size variation.

Mesoporous nanomaterials with unique architectures are also applied in fields other than catalysis, energy conversion and biomedicines. In two separated works, Lan et al. and Fang et al. both utilized 2D mesoporous heterostructures for high performance energy storages. ${ }^{47,48}$ Ren et al. architecture orthogonally assembled 3D cross-stacked metal oxide semiconducting nanowires, which showed brilliant gas sensing properties. ${ }^{49}$ Zhu et al. fabricated mesoporous tungsten oxides with crystalline frameworks for highly sensitive pathogen detections. ${ }^{50}$ Liu et al. demonstrated that mesoporous silica membranes with large vertical mesochannels can be used for efficient nanosize-based separations. ${ }^{51}$ Overall, mesoporous nanomaterials with unique architectures have exciting application potentials in an extremely large range of fields.

\section{CONCLUSIONS AND PERSPECTIVE}

Great progress has been achieved in the interfacial assembly directed unique mesoporous nanomaterials. A series of functional mesoporous nanomaterials with unique architectures have been successfully prepared in recent years, ranging from symmetrical to asymmetric structures. Strategies used to architecture these unique mesoporous nanomaterials include interfacial assembly on various interfaces, such as liquidliquid, solid-liquid, solid-solid, etc., as well as tuning the interfacial assembly behaviors based on interfacial energy, inner tension, and so on. These mesoporous nanomaterials obtained from interfacial assembly have precisely manipulated architectures, exhibiting excellent performance in catalysis, energy conversion and storage, biomedicine and so on. Albeit great progress, there are still numerous difficulties to be tackled in the future, typically, in designing special mesoporous architectures and establishing the structure-function relationship, related theory, etc. in the applications. Though hindering developments, these obstacles also point out the future road of the field.

1. Direct observation and understanding of the singlemicelle based interfacial-assembly process: Recently, the concept of "single-micelle" has aroused a wide range of attention. By considering the micelles as independent entities, researchers are able to obtain brand new understandings of the micelles' assembly process. However, observing the single-micelle assembly process would require the assist of advanced technologies, such as cryo-TEM, environmental-SEM, in situ X-ray diffractions and so on. In-situ observation and understanding the assembly process of the micelles at the interface may endow more precise control of the micelles' assembly behaviors.

2. Precise manipulation of mesopore parameters: As noted above, properties such as size, crystallinity, mesostructure, pore channels, and surface physicochemistry may significantly affect the overall performance of mesoporous nanomaterials. Size plays a crucial role in nanomaterials' applications. Currently, some of the fabricated mesoporous nanoparticles are too large, making them a bit unsuitable for applications; for example, the mesoporous $\mathrm{TiO}_{2}$ microspheres are of micrometer size, limiting their surface area for interacting with environments. Also, a sub-100 nm size is usually preferred for the mesoporous nanoparticles designed for biological applications, yet most of present mesoporous nanoparticles do not fit in this range. Besides, it is quite important to precisely tune mesopore parameters of mesoporous nanomaterials. Especially, when there are multiple mesoporous compartments in one nanocomposite, the independent manipulation of the mesoporous parameters in each compartment would be crucial.

3. Asymmetric mesoporous nanoparticles with precisely controllable architectures: First, presently obtained nanomaterials are mostly dual compartments, which is due to limited synthetic methods. For example, the anisotropic assembly of PMO is based on the surface tension between PMO and another material. Thus, this methodology cannot function for the repeated growth of PMOs on PMOs with similar chemical properties. ${ }^{18}$ Therefore, the investigation on anisotropic assembly of some other mesoporous materials is essential for the construction of more complex multicompartment mesoporous nanoparticles. On the other hand, the precise manipulation of the nucleation sites (number, location, orientation, etc.) is also crucial. For most of the reported asymmetric nanocomposite, the relative location of the newly formed compartment is rather random. If the location of anisotropic assembly can be precisely controlled, the different mesoporous compartments can be used as modular blocks for the fabrication of truly multicompartment mesoporous nanomaterials. The last but not the least, the superassembly of the asymmetric nanoparticles is still a great challenge. The secondary assembly of the asymmetric mesoporous nanoparticles into 1D fiber, 2D film, 3D superstructure, etc. is crucial for the manufacture of devices in the practical use.

4. Novel applications based on advanced architectures: Albeit great progress has been made in the synthesis of mesoporous nanomaterials with unique architectures, the structure-function relationship and related theory in the applications are yet to be established. For example, electrochemical applications require the coexistence of both mesopores (for high surface area) as well as high crystallinity (for high electron transfer rate), thus the field longs for highly crystallized mesoporous materials. Interactions between nanomaterials and biological hosts varies with the morphology and surface chemistry of 
nanoparticles. Deeper investigations and understanding the dominance factors of the interaction may tell researchers what architectures are actually needed for enhanced nanobio interactions.

\section{AUTHOR INFORMATION}

\section{Corresponding Authors}

Xiaomin Li - Department of Chemistry, Laboratory of Advanced Materials and Shanghai Key Laboratory of Molecular Catalysis and Innovative Materials, State Key Laboratory of Molecular Engineering of Polymers, Collaborative Innovation Center of Chemistry for Energy Materials (2011-iChEM), Fudan University, Shanghai 200433, P. R. China; ○ orcid.org/0000-0001-6056-6928; Email: lixm@ fudan.edu.cn

Dongyuan Zhao - Department of Chemistry, Laboratory of Advanced Materials and Shanghai Key Laboratory of Molecular Catalysis and Innovative Materials, State Key Laboratory of Molecular Engineering of Polymers, Collaborative Innovation Center of Chemistry for Energy Materials (2011-iChEM), Fudan University, Shanghai 200433, P. R. China; ○ orcid.org/0000-0001-8440-6902; Email: dyzhao@ fudan.edu.cn

\section{Authors}

Tiancong Zhao - Department of Chemistry, Laboratory of Advanced Materials and Shanghai Key Laboratory of Molecular Catalysis and Innovative Materials, State Key Laboratory of Molecular Engineering of Polymers, Collaborative Innovation Center of Chemistry for Energy Materials (2011iChEM), Fudan University, Shanghai 200433, P. R. China

Liang Chen - Department of Chemistry, Laboratory of Advanced Materials and Shanghai Key Laboratory of Molecular Catalysis and Innovative Materials, State Key Laboratory of Molecular Engineering of Polymers, Collaborative Innovation Center of Chemistry for Energy Materials (2011iChEM), Fudan University, Shanghai 200433, P. R. China

Runfeng Lin - Department of Chemistry, Laboratory of Advanced Materials and Shanghai Key Laboratory of Molecular Catalysis and Innovative Materials, State Key Laboratory of Molecular Engineering of Polymers, Collaborative Innovation Center of Chemistry for Energy Materials (2011iChEM), Fudan University, Shanghai 200433, P. R. China

Pengfei Zhang - Department of Chemistry, Laboratory of Advanced Materials and Shanghai Key Laboratory of Molecular Catalysis and Innovative Materials, State Key Laboratory of Molecular Engineering of Polymers, Collaborative Innovation Center of Chemistry for Energy Materials (2011iChEM), Fudan University, Shanghai 200433, P. R. China

Kun Lan - Department of Chemistry, Laboratory of Advanced Materials and Shanghai Key Laboratory of Molecular Catalysis and Innovative Materials, State Key Laboratory of Molecular Engineering of Polymers, Collaborative Innovation Center of Chemistry for Energy Materials (2011-iChEM), Fudan University, Shanghai 200433, P. R. China

Wei Zhang - Department of Chemistry, Laboratory of Advanced Materials and Shanghai Key Laboratory of Molecular Catalysis and Innovative Materials, State Key Laboratory of Molecular Engineering of Polymers, Collaborative Innovation Center of Chemistry for Energy Materials (2011-iChEM), Fudan University, Shanghai 200433, P. R. China

Complete contact information is available at: https://pubs.acs.org/10.1021/accountsmr.0c00028

\section{Author Contributions}

The manuscript was written through contributions of all authors. All authors have given approval to the final version of the manuscript.

\section{Funding}

The work was supported by the National Key R\&D Program of China (2018YFA0209401), National Natural Science Foundation of China (21875043, 22075049, 21733003, 21701027, 51961145403), Key Basic Research Program of Science and Technology Commission of Shanghai Municipality (17JC1400100), Natural Science Foundation of Shanghai (18ZR1404600), and Shanghai Rising-Star Program (20QA1401200).

\section{Notes}

The authors declare no competing financial interest.

\section{Biographies}

Tiancong Zhao was born in Changchun, Jilin, in April 1993. He received his Bachelor Degree at Fudan University in 2016. He is now studying for a doctorate at Prof. Dongyuan Zhao's group in Fudan University. His current research mainly focuses on design and fabrication of functional nanomaterials and their applications in biological fields.

Liang Chen received his Ph.D. from Donghua University in 2018. He is currently a Postdoctoral Fellow at Laboratory of Advanced Materials, Fudan University. His research interests are focused on the design and synthesis of organic/inorganic functional nanomaterials for biomedical applications, including drug delivery, diagnostic imaging, regenerative medicine, and multimodal disease theranostics.

Runfeng Lin joined Prof. Zhao's group in Fudan University to pursue his Ph.D. degree. His current research interests focus on the development of carbon-silica hybrid materials for applications and simulation in drug delivery system.

Pengfei Zhang received his Bachelor degree from Wuhan University of Technology in 2015. Now, he is a Ph.D. candidate in Laboratory of Advanced Materials, Fudan University. His research interests focus on the synthesis of mesoporous semiconductors for energy storage.

Kun Lan received his Ph.D. in 2020 from Fudan University. He is currently a postdoctoral fellow with Prof. Dongyuan Zhao in Laboratory of Advanced Materials, Fudan University. His current research interests focus on the precision syntheses of crystalline mesostructured materials as energy-related fundamental science.

Wei Zhang received his Ph.D. from Fudan University in 2020. Now, he is a postdoctoral fellow with Prof. Dongyuan Zhao in the Laboratory of Advanced Materials, Fudan University. His current research interests focus on the rational design of mesoporous nanocomposites for energy storage and conversion, and catalysis.

Xiaomin Li received his Ph.D. in 2014 from Fudan University followed by 2 years postdoctoral experience in Fudan University and Griffith University. He joined Chemistry Department of Fudan University in 2016. His current research interests include the development of multifunctional nanostructured materials and nanotechnology for applications in bioimaging, nanomedicine, analysis, and drug delivery.

Dongyuan Zhao received his B.S. (1984) and Ph.D. (1990) from Jilin University. Then, he joined the Weizmann Institute of Science (1993-1994), University of Houston (1995-1996), and University 
of California at Santa Barbara (1996-1998) for postdoctoral research. $\mathrm{He}$ is now a Professor in the Department of Chemistry at Fudan University. He was elected as an academician of the Chinese Academy of Science in 2007 and a member of The World Academy of Science in 2010. His research interests include the synthesis of mesoporous materials for catalysis, energy, environmental, and biomedical applications.

\section{REFERENCES}

(1) Wan, Y.; Zhao, D. On the controllable soft-templating approach to mesoporous silicates. Chem. Rev. 2007, 107, 2821-2860.

(2) Zhao, T. C.; Elzatahry, A.; Li, X. M.; Zhao, D. Y. Single-micelledirected synthesis of mesoporous materials. Nat. Rev. Mater. 2019, 4, 775-791.

(3) Kresge, C.; Leonowicz, M.; Roth, W.; Vartuli, J.; Beck, J. Ordered mesoporous molecular sieves synthesized by a liquid-crystal template mechanism. Nature 1992, 359 (6397), 710-712.

(4) Zhao, D. Y.; Feng, J.; Huo, Q.; Melosh, N.; Fredrickson, G.; Chmelka, B.; Stucky, G. D. Triblock copolymer syntheses of mesoporous silica with periodic 50 to 300 angstrom pores. Science 1998, 279 (5350), 548-552.

(5) Li, C.; Iqbal, M.; Lin, J.; Luo, X.; Jiang, B.; Malgras, V.; Wu, K.; Kim, J.; Yamauchi, Y. Electrochemical deposition: an advanced approach for templated synthesis of nanoporous metal architectures. Acc. Chem. Res. 2018, 51 (8), 1764-1773.

(6) Kankala, R.; Han, Y.; Na, J.; Lee, C.; Sun, Z.; Wang, S.; Kimura, T.; Ok, Y.; Yamauchi, Y.; Chen, A.; Wu, K. Nanoarchitectured structure and surface biofunctionality of mesoporous silica nanoparticles. Adv. Mater. 2020, 32 (23), No. e1907035.

(7) Li, C.; Iqbal, M.; Jiang, B.; Wang, Z.; Kim, J.; Nanjundan, A.; Whitten, A.; Wood, K.; Yamauchi, Y. Pore-tuning to boost the electrocatalytic activity of polymeric micelle-templated mesoporous Pd nanoparticles. Chem. Sci. 2019, 10 (14), 4054-4061.

(8) Qiu, P.; Ma, B.; Hung, C. T.; Li, W.; Zhao, D. Spherical mesoporous materials from single to multilevel architectures. Acc. Chem. Res. 2019, 52, 2928-2938.

(9) Parlett, C. M.; Isaacs, M. A.; Beaumont, S. K.; Bingham, L. M.; Hondow, N. S.; Wilson, K.; Lee, A. F. Spatially orthogonal chemical functionalization of a hierarchical pore network for catalytic cascade reactions. Nat. Mater. 2016, 15, 178-182.

(10) Jiang, B.; Guo, Y.; Kim, J.; Whitten, A.; Wood, K.; Kani, K.; Rowan, A.; Henzie, J.; Yamauchi, Y. Mesoporous metallic Iridium nanosheets. J. Am. Chem. Soc. 2018, 140 (39), 12434-12441.

(11) Li, W.; Liu, J.; Zhao, D. Y. Mesoporous materials for energy conversion and storage devices. Nat. Rev. Mater. 2016, 1, 16023.

(12) Lv, H.; Xu, D.; Sun, L.; Henzie, J.; Lopes, A.; Gu, Q.; Yamauchi, Y.; Liu, B. Asymmetric multimetallic mesoporous nanospheres. Nano Lett. 2019, 19 (5), 3379-3385.

(13) Zhao, T.; Chen, L.; Li, Q.; Li, X. M. Near-infrared light triggered drug release from mesoporous silica nanoparticles. J. Mater. Chem. B 2018, 6 (44), 7112-7121.

(14) Li, W.; Yue, Q.; Deng, Y.; Zhao, D. Ordered mesoporous materials based on interfacial assembly and engineering. Adv. Mater. 2013, 25, 5129-5152.

(15) Wang, W.; Wang, P.; Tang, X.; Elzatahry, A. A.; Wang, S.; AlDahyan, D.; Zhao, M.; Yao, C.; Hung, C. T.; Zhu, X.; Zhao, T.; Li, X.; Zhang, F.; Zhao, D. Facile synthesis of uniform virus-like mesoporous silica nanoparticles for enhanced cellular internalization. ACS Cent. Sci. 2017, 3, 839-846.

(16) Liu, Y.; Lan, K.; Li, S.; Liu, Y.; Kong, B.; Wang, G.; Zhang, P.; Wang, R.; He, H.; Ling, Y.; Al-Enizi, A. M.; Elzatahry, A. A.; Cao, Y.; Chen, G.; Zhao, D. Constructing three-dimensional mesoporous bouquet-posy-like $\mathrm{TiO}_{2}$ superstructures with radially oriented mesochannels and single-crystal walls. J. Am. Chem. Soc. 2017, 139, 517-526.

(17) Teng, Z.; Su, X.; Zheng, Y.; Zhang, J.; Liu, Y.; Wang, S.; Wu, J.; Chen, G.; Wang, J.; Zhao, D.; Lu, G. A facile multi-interface transformation approach to monodisperse multiple-shelled periodic mesoporous organosilica hollow spheres. J. Am. Chem. Soc. 2015, 137, $7935-7944$.

(18) Li, X.; Zhou, L.; Wei, Y.; El-Toni, A. M.; Zhang, F.; Zhao, D. Anisotropic growth-induced synthesis of dual-compartment Janus mesoporous silica nanoparticles for bimodal triggered drugs delivery. J. Am. Chem. Soc. 2014, 136 (42), 15086-15092.

(19) Zhao, T. C.; Chen, L.; Wang, P.; Li, B.; Lin, R.; Abdulkareem Al-Khalaf, A.; Hozzein, W. N.; Zhang, F.; Li, X. M.; Zhao, D. Y. Surface-kinetics mediated mesoporous multipods for enhanced bacterial adhesion and inhibition. Nat. Commun. 2019, 10, 4387.

(20) Fang, Y.; Lv, Y.; Gong, F.; Wu, Z.; Li, X.; Zhu, H.; Zhou, L.; Yao, C.; Zhang, F.; Zheng, G.; Zhao, D. Interface tension-induced synthesis of monodispersed mesoporous carbon hemispheres. J. Am. Chem. Soc. 2015, 137, 2808-2811.

(21) Shen, D.; Yang, J.; Li, X.; Zhou, L.; Zhang, R.; Li, W.; Chen, L.; Wang, R.; Zhang, F.; Zhao, D. Biphase stratification approach to three-dimensional dendritic biodegradable mesoporous silica nanospheres. Nano Lett. 2014, 14, 923-932.

(22) Yang, T.; Wei, L.; Jing, L.; Liang, J.; Zhang, X.; Tang, M.; Monteiro, M. J.; Chen, Y. I.; Wang, Y.; Gu, S.; Zhao, D.; Yang, H.; Liu, J.; Lu, G. Q. M. Dumbbell-shaped bi-component mesoporous janus solid nanoparticles for biphasic interface catalysis. Angew. Chem., Int. Ed. 2017, 56, 8459-8463.

(23) Shen, D.; Chen, L.; Yang, J.; Zhang, R.; Wei, Y.; Li, X.; Li, W.; Sun, Z.; Zhu, H.; Abdullah, A. M.; Al-Enizi, A.; Elzatahry, A. A.; Zhang, F.; Zhao, D. Ultradispersed palladium nanoparticles in threedimensional dendritic mesoporous silica nanospheres: toward active and stable heterogeneous catalysts. ACS Appl. Mater. Interfaces 2015, 7, 17450-17459.

(24) Wang, X. Q.; Zhang, Y.; Luo, W.; Elzatahry, A. A.; Cheng, X. W.; Alghamdi, A.; Abdullah, A. M.; Deng, Y. H.; Zhao, D. Y. Synthesis of ordered mesoporous silica with tunable morphologies and pore sizes via a nonpolar solvent-assisted stober method. Chem. Mater. 2016, 28, 2356-2362.

(25) Fang, Y.; Gu, D.; Zou, Y.; Wu, Z.; Li, F.; Che, R.; Deng, Y.; Tu, B.; Zhao, D. A low-concentration hydrothermal synthesis of biocompatible ordered mesoporous carbon nanospheres with tunable and uniform size. Angew. Chem., Int. Ed. 2010, 49, 7987-7991.

(26) Liu, J.; Yang, T.; Wang, D.-W.; Lu, G. Q.; Zhao, D.; Qiao, S. Z. A facile soft-template synthesis of mesoporous polymeric and carbonaceous nanospheres. Nat. Commun. 2013, 4, 2798.

(27) Liu, Y.; Che, R.; Chen, G.; Fan, J.; Sun, Z.; Wu, Z.; Wang, M.; Li, B.; Wei, J.; Wei, Y.; Wang, G.; Guan, G.; Elzatahry, A. A.; Bagabas, A. A.; Al-Enizi, A. M.; Deng, Y.; Peng, H.; Zhao, D. Radially oriented mesoporous $\mathrm{TiO}_{2}$ microspheres with single-crystal-like anatase walls for high-efficiency optoelectronic devices. Sci. Adv. 2015, 1, No. e1500166.

(28) Yue, Q.; Li, J.; Luo, W.; Zhang, Y.; Elzatahry, A. A.; Wang, X.; Wang, C.; Li, W.; Cheng, X.; Alghamdi, A.; Abdullah, A. M.; Deng, Y.; Zhao, D. An interface coassembly in biliquid phase: toward core-shell magnetic mesoporous silica microspheres with tunable pore size. J. Am. Chem. Soc. 2015, 137, 13282-13289.

(29) Peng, L.; Hung, C. T.; Wang, S.; Zhang, X.; Zhu, X.; Zhao, Z.; Wang, C.; Tang, Y.; Li, W.; Zhao, D. Versatile nanoemulsion assembly approach to synthesize functional mesoporous carbon nanospheres with tunable pore sizes and architectures. J. Am. Chem. Soc. 2019, 141, 7073-7080.

(30) Lan, K.; Liu, Y.; Zhang, W.; Liu, Y.; Elzatahry, A.; Wang, R.; Xia, Y.; Al-Dhayan, D.; Zheng, N.; Zhao, D. Uniform ordered twodimensional mesoporous $\mathrm{TiO}_{2}$ nanosheets from hydrothermalinduced solvent-confined monomicelle assembly. J. Am. Chem. Soc. 2018, 140, 4135-4143.

(31) Li, X.; Guo, Z.; Zhao, T.; Lu, Y.; Zhou, L.; Zhao, D.; Zhang, F. Filtration shell mediated power density independent orthogonal excitations-emissions upconversion luminescence. Angew. Chem., Int. Ed. 2016, 55, 2464-2469.

(32) Yang, J.; Shen, D.; Zhou, L.; Li, W.; Li, X.; Yao, C.; Wang, R.; El-Toni, A. M.; Zhang, F.; Zhao, D. Spatially confined fabrication of core-shell gold nanocages@mesoporous silica for near-infrared 
controlled photothermal drug release. Chem. Mater. 2013, 25, 30303037.

(33) Li, W.; Yang, J.; Wu, Z.; Wang, J.; Li, B.; Feng, S.; Deng, Y.; Zhang, F.; Zhao, D. A versatile kinetics-controlled coating method to construct uniform porous $\mathrm{TiO}_{2}$ shells for multifunctional core-shell structures. J. Am. Chem. Soc. 2012, 134, 11864-11867.

(34) Wang, M.; Sun, Z.; Yue, Q.; Yang, J.; Wang, X.; Deng, Y.; Yu, C.; Zhao, D. An interface-directed coassembly approach to synthesize uniform large-pore mesoporous silica spheres. J. Am. Chem. Soc. 2014, 136, 1884-1892.

(35) Zhang, P.; Tian, Z.; Hung, C.; Liu, Y.; Jia, B.; Lan, K.; Kong, B.; Huang, F.; Mai, L.; Zhao, D. Y. Branched mesoporous $\mathrm{TiO}_{2}$ mesocrystals by epitaxial assembly of micelles for photocatalysis. Cell Report Physical Science 2020, 1, 100081.

(36) Yue, Q.; Zhang, Y.; Jiang, Y.; Li, J.; Zhang, H.; Yu, C.; Elzatahry, A. A.; Alghamdi, A.; Deng, Y.; Zhao, D. Nanoengineering of core-shell magnetic mesoporous microspheres with tunable surface roughness. J. Am. Chem. Soc. 2017, 139, 4954-4961.

(37) Yue, Q.; Li, J.; Zhang, Y.; Cheng, X.; Chen, X.; Pan, P.; Su, J.; Elzatahry, A. A.; Alghamdi, A.; Deng, Y.; Zhao, D. Plasmolysisinspired nanoengineering of functional yolk-shell microspheres with magnetic core and mesoporous silica shell. J. Am. Chem. Soc. 2017, 139, 15486-15493.

(38) Lan, K.; Xia, Y.; Wang, R. C.; Zhao, Z. W.; Zhang, W.; Zhang, X. M.; Elzatahry, A.; Zhao, D. Y. Confined interfacial monomicelle assembly for precisely controlled coating of single-layered titania mesopores. Matter 2019, 1, 527-538.

(39) Walther, A.; Muller, A. H. Janus particles: synthesis, selfassembly, physical properties, and applications. Chem. Rev. 2013, 113, 5194-5261.

(40) Li, X.; Zhou, L.; Wei, Y.; El-Toni, A. M.; Zhang, F.; Zhao, D. Anisotropic encapsulation-induced synthesis of asymmetric singlehole mesoporous nanocages. J. Am. Chem. Soc. 2015, 137, 59035906.

(41) Li, X.; Zhao, T.; Lu, Y.; Wang, P.; El-Toni, A. M.; Zhang, F.; Zhao, D. Degradation-restructuring induced anisotropic epitaxial growth for fabrication of asymmetric diblock and triblock mesoporous nanocomposites. Adv. Mater. 2017, 29, 1701652.

(42) Wang, W. X.; Wang, P. Y.; Chen, L.; Zhao, M. Y.; Hung, C. T.; Yu, C. Z.; Al-Khalaf, A. A.; Hozzein, W. N.; Zhang, F.; Li, X. M.; Zhao, D. Y. Engine-trailer-structured nanotrucks for efficient nano-bio interactions and bioimaging-guided drug delivery. Chem. 2020, 6, $1097-1112$.

(43) Lan, K.; Wang, R. C.; Zhang, W.; Zhao, Z. W.; Elzatahry, A.; Zhang, X. M.; Liu, Y.; Al-Dhayan, D.; Xia, Y. Y.; Zhao, D. Y. Mesoporous $\mathrm{TiO}_{2}$ microspheres with precisely controlled crystallites and architectures. Chem 2018, 4, 2436-2450.

(44) Zhao, T.; Zhu, X.; Hung, C. T.; Wang, P.; Elzatahry, A.; AlKhalaf, A. A.; Hozzein, W. N.; Zhang, F.; Li, X.; Zhao, D. Spatial isolation of carbon and silica in a single janus mesoporous nanoparticle with tunable amphiphilicity. J. Am. Chem. Soc. 2018, 140, 10009-10015.

(45) Teng, Z.; Wang, C.; Tang, Y.; Li, W.; Bao, L.; Zhang, X.; Su, X.; Zhang, F.; Zhang, J.; Wang, S.; Zhao, D.; Lu, G. Deformable hollow periodic mesoporous organosilica nanocapsules for significantly improved cellular uptake. J. Am. Chem. Soc. 2018, 140, 1385-1393.

(46) Zhang, W.; He, H.; Tian, Y.; Lan, K.; Liu, Q.; Wang, C.; Liu, Y.; Elzatahry, A.; Che, R.; Li, W.; Zhao, D. Synthesis of uniform ordered mesoporous $\mathrm{TiO}_{2}$ microspheres with controllable phase junctions for efficient solar water splitting. Chem. Sci. 2019, 10, 1664-1670.

(47) Fang, Y.; Lv, Y.; Gong, F.; Elzatahry, A. A.; Zheng, G.; Zhao, D. Synthesis of 2D-mesoporous-carbon/ $\mathrm{MoS}_{2}$ heterostructures with well-defined interfaces for high-performance lithium-ion batteries. Adv. Mater. 2016, 28 (42), 9385-9390.

(48) Lan, K.; Wei, Q.; Wang, R.; Xia, Y.; Tan, S.; Wang, Y.; Elzatahry, A.; Feng, P.; Mai, L.; Zhao, D. Two-dimensional mesoporous heterostructure delivering superior pseudocapacitive sodium storage via bottom-up monomicelle assembly. J. Am. Chem. Soc. 2019, 141 (42), 16755-16762.
(49) Ren, Y.; Zou, Y.; Liu, Y.; Zhou, X.; Ma, J.; Zhao, D. Y.; Wei, G.; Ai, Y.; Xi, S.; Deng, Y. Synthesis of orthogonally assembled 3D crossstacked metal oxide semiconducting nanowires. Nat. Mater. 2020, 19, 203-211.

(50) Zhu, Y.; Zhao, Y.; Ma, J.; Cheng, X.; Xie, J.; Xu, P.; Liu, H.; Liu, H.; Zhang, H.; Wu, M.; Elzatahry, A. A.; Alghamdi, A.; Deng, Y.; Zhao, D. mesoporous Tungsten oxides with crystalline framework for highly sensitive and selective detection of foodborne pathogens. J. Am. Chem. Soc. 2017, 139 (30), 10365-10373.

(51) Liu, Y.; Shen, D.; Chen, G.; Elzatahry, A. A.; Pal, M.; Zhu, H.; Wu, L.; Lin, J.; Al-Dahyan, D.; Li, W.; Zhao, D. Mesoporous silica thin membranes with large vertical mesochannels for nanosize-based separation. Adv. Mater. 2017, 29 (35), 1702274. 